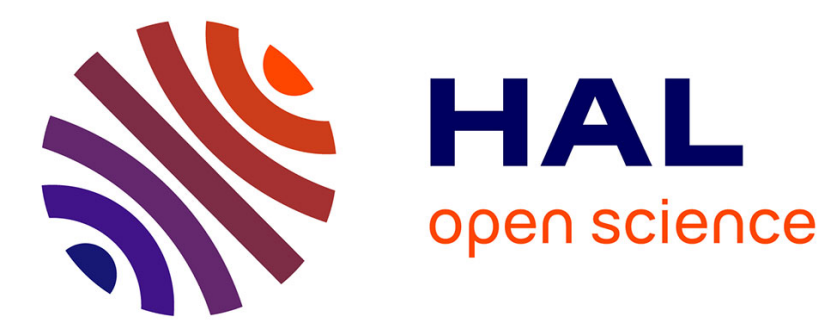

\title{
Deterministic mean field games with control on the acceleration
}

\author{
Yves Achdou, Paola Mannucci, Claudio Marchi, Nicoletta Tchou
}

\section{To cite this version:}

Yves Achdou, Paola Mannucci, Claudio Marchi, Nicoletta Tchou. Deterministic mean field games with control on the acceleration. Nonlinear Differential Equations and Applications, 2020, 27 (33), 10.1007/s00030-020-00634-y . hal-02265051v3

\section{HAL Id: hal-02265051 \\ https://hal.science/hal-02265051v3}

Submitted on 23 Jun 2020

HAL is a multi-disciplinary open access archive for the deposit and dissemination of scientific research documents, whether they are published or not. The documents may come from teaching and research institutions in France or abroad, or from public or private research centers.
L'archive ouverte pluridisciplinaire HAL, est destinée au dépôt et à la diffusion de documents scientifiques de niveau recherche, publiés ou non, émanant des établissements d'enseignement et de recherche français ou étrangers, des laboratoires publics ou privés. 


\title{
Deterministic mean field games with control on the acceleration
}

\author{
Yves Achdou *Paola Mannucci† \\ Claudio Marchi $\stackrel{\ddagger}{\ddagger}$ Nicoletta Tchou ${ }^{\S}$
}

June 23, 2020

\begin{abstract}
In the present work, we study deterministic mean field games (MFGs) with finite time horizon in which the dynamics of a generic agent is controlled by the acceleration. They are described by a system of PDEs coupling a continuity equation for the density of the distribution of states (forward in time) and a Hamilton-Jacobi (HJ) equation for the optimal value of a representative agent (backward in time).

The state variable is the pair $(x, v) \in \mathbb{R}^{N} \times \mathbb{R}^{N}$ where $x$ stands for the position and $v$ stands for the velocity. The dynamics is often referred to as the double integrator. In this case, the Hamiltonian of the system is neither strictly convex nor coercive, hence the available results on MFGs cannot be applied. Moreover, we will assume that the Hamiltonian is unbounded w.r.t. the velocity variable $v$. We prove the existence of a weak solution of the MFG system via a vanishing viscosity method and we characterize the distribution of states as the image of the initial distribution by the flow associated with the optimal control.
\end{abstract}

Keywords: Mean field games, first order Hamilton-Jacobi equations, double integrator, non-coercive Hamiltonian.

2010 AMS Subject classification: 35F50, 35Q91, 49K20, 49L25.

\section{Introduction}

The theory of mean field games (MFGs for short) is more and more investigated since the pioneering works $[24,25,26]$ of Lasry and Lions: it aims at studying the asymptotic behaviour of differential games (Nash equilibria) as the number of agents tends to infinity. In the present work, we study deterministic mean field games with finite time horizon in which the dynamics of a generic agent is controlled by the acceleration. They are described by a system of PDEs coupling a continuity equation for the density of the distribution of states (forward in time) and a Hamilton-Jacobi (HJ) equation for the optimal value of a representative agent (backward in time). The state variable is the pair $(x, v) \in \mathbb{R}^{N} \times \mathbb{R}^{N}$ where $x$ stands for the position and $v$ stands for the velocity.

\footnotetext{
*Université de Paris, Laboratoire Jacques-Louis Lions (LJLL), F-75013 Paris, France, achdou@ljll-univparis-diderot.fr

†Dipartimento di Matematica "Tullio Levi-Civita”, Università di Padova, mannucci@math.unipd.it

${ }^{\ddagger}$ Dipartimento di Ingegneria dell’Informazione, Università di Padova, claudio.marchi@unipd.it

§Univ Rennes, CNRS, IRMAR - UMR 6625, F-35000 Rennes, France, nicoletta.tchou@univ-rennes1.fr
} 
The systems of PDEs are of the form

$(1.1)$

$$
\left\{\begin{array}{lll}
(i) & -\partial_{t} u-v \cdot D_{x} u+H\left(x, v, D_{v} u\right)-F[m(t)](x, v)=0 & \text { in } \mathbb{R}^{2 N} \times(0, T) \\
(i i) & \partial_{t} m+v \cdot D_{x} m-\operatorname{div}_{v}\left(D_{p_{v}} H\left(x, v, D_{v} u\right) m\right)=0 & \text { in } \mathbb{R}^{2 N} \times(0, T) \\
(\text { iii }) & m(x, v, 0)=m_{0}(x, v), u(x, v, T)=G[m(T)](x, v), & \text { on } \mathbb{R}^{2 N}
\end{array}\right.
$$

where $T$ is a positive real number, $u=u(x, v, t), m=m(x, v, t),(x, v) \in \mathbb{R}^{2 N}, t \in(0, T)$ and $H$ is defined by

$$
H\left(x, v, p_{v}\right)=\max _{\alpha \in \mathbb{R}^{N}}\left(-\alpha p_{v}-l(x, v, \alpha)\right) .
$$

We take $F$ and $G$ strongly regularizing and we assume that the running cost has the form $l(x, v, \alpha)=l(x, v)+\frac{1}{2}|\alpha|^{2}+\frac{1}{2}|v|^{2}$, where $(x, v) \mapsto l(x, v)$ is a bounded and $C^{2}$-bounded function.

Formally, systems of this form arise when the dynamics of the generic player is described by a double integrator:

$$
\left\{\begin{array}{rlrl}
\xi^{\prime}(s) & =\eta(s), & & s \in(t, T), \\
\eta^{\prime}(s) & =\alpha(s), & & s \in(t, T), \\
\xi(t) & =x & \\
\eta(t) & =v &
\end{array}\right.
$$

and when the control law belongs to the space of the measurable functions with values in $\mathbb{R}^{N}$ and is chosen in order to minimize the cost

$$
J_{t}:=J_{t}(\xi, \eta, \alpha)=\int_{t}^{T} l(\xi(s), \eta(s), \alpha(s))+F[m(s)](\xi(s), \eta(s)) d s+G[m(T)](\xi(T), \eta(T)) .
$$

To summarize, the main features of this model are:

1. The control $\alpha$ is only involved in the dynamics of the second component of the state variable, see (1.3).

2. The running cost has the form

$$
l(\xi, \eta, \alpha)=l(\xi, \eta)+\frac{1}{2}|\eta|^{2}+\frac{1}{2}|\alpha|^{2},
$$

where $(\xi, \eta) \mapsto l(\xi, \eta)$ is a bounded $C^{2}$ function, thus the former is unbounded w.r.t. the variable $\eta$. Note that $|\eta|^{2}$ stands for a kinetic energy, whereas the term $|\alpha|^{2}$ is a penalty for large accelerations. Note also that the results of the present paper hold for a fairly large class of generalizations of (1.5).

3. Setting $f(\xi, \eta, \alpha)=(\eta, \alpha)$, the Hamiltonian associated to the control problem of a generic player is

$$
\mathcal{H}(\xi, \eta, p)=\max _{\alpha \in \mathbb{R}^{N}}\{-p \cdot f(\xi, \eta, \alpha)-l(\xi, \eta, \alpha)\}=-p_{x} \cdot \eta+H\left(\xi, \eta, p_{v}\right),
$$

where $p=\left(p_{x}, p_{v}\right)$ and $H$ is defined in (1.2). The Hamilton $\mathcal{H}$ is neither strictly convex nor coercive with respect to $p=\left(p_{x}, p_{v}\right)$. Hence the available results on the regularity of the value function $u$ of the associated optimal control problem ([11], [18], [12]) and on the existence of a solution of the MFG system ([12]) cannot be applied. 
We recently learnt that a similar type of mean field games has been studied in [10], independently, at the same time, and with different techniques. To the best of our knowledge, these systems have not been investigated elsewhere.

The main results of the present work are the existence of a solution of (2.1) and a characterization of the distribution of states $m$. In order to establish the representation formula for $m$, we use some ideas introduced by P-L Lions in his lectures at Collège de France (2012) (see [28, 12]), some results proved in [15, 13], and the superposition principle [2, Theorem 8.2.1]. These methods rely on optimal control theory, in particular on optimal synthesis results. In our setting, the lack of coercivity of $\mathcal{H}$ makes it impossible to directly apply the arguments of [12, Sect. 4.1], in particular a contraction property of the flow associated to the dynamics (see [12, Lemma 4.13]). However, the superposition principle and suitable optimal synthesis results will be used to characterize $m$ as the image of the initial distribution by the optimal flow associated with the Hamilton-Jacobi equation. By standard techniques for monotone operators (see Lasry and Lions [26]), we also obtain the uniqueness of the solution under classical assumptions.

The superposition principle has already been used in a different approach, for instance in the articles of Cardaliaguet [14], Cardaliaguet, Mészáros and Santambrogio [16] and Orrieri, Porretta and Savaré [30]. In these works, the authors tackle the MFG systems of the first order using a variational approach based on two optimization problems in duality, under suitable assumptions. Then, using the superposition principle, they are able to describe the solution to the continuity equation arising in the optimality conditions of the latter optimization problem by means of a measure on the space of continuous paths. This measure is concentrated on the set of minimizing curves for the optimal control problem underlying the Hamilton-Jacobi equation.

A similar approach to the one of the present paper was recently proposed for a class of non-coercive MFG when the generic player has some "forbidden direction" (see [29]), more precisely when, in the two dimensional case, the dynamics is of the form: $x_{1}^{\prime}=\alpha_{1}$, $x_{2}^{\prime}=h\left(x_{1}\right) \alpha_{2}$ and $h\left(x_{1}\right)$ may vanish.

In a near future, we plan to tackle mean field games with control on the acceleration and with constraints (for MFGs with state constraints we refer to $[1,7,8,9]$ ).

The paper is organized as follows. In Section 2, we list our assumptions, give the definition of (weak) solution to system (2.1) and state the existence and uniqueness results for the latter. In Section 3, we obtain some regularity properties for the solution $u$ of the Hamilton-Jacobi equation (2.1)-(i) with $m$ fixed. These properties, combined with the uniqueness of the optimal trajectories of the associated control problem, will be crucial for proving the main theorem. In Section 4, we study the continuity equation (2.1)-(ii). An important ingredient is the vanishing viscosity method that is used to characterize its solution. Finally, Section 5 is devoted to the proofs of the main Theorem 2.1 on the existence of a solution and of Proposition 2.1 on its uniqueness. In the Appendix, following a suggestion of the referee, we establish the existence and the uniqueness of the solution to the corresponding second order MFG system as a byproduct of the estimates needed for the vanishing viscosity limit. 


\section{Assumptions and main results}

We consider the running cost $l(x, v, \alpha)$ of the form $l(x, v, \alpha)=l(x, v)+\frac{1}{2}|\alpha|^{2}+\frac{1}{2}|v|^{2}$. Then system (1.1) can be written

$$
\left\{\begin{array}{lll}
(i) & -\partial_{t} u-v \cdot D_{x} u+\frac{1}{2}\left|D_{v} u\right|^{2}-\frac{1}{2}|v|^{2}-l(x, v)-F[m](x, v)=0, & \text { in } \mathbb{R}^{2 N} \times(0, T), \\
(i i) & \partial_{t} m+v \cdot D_{x} m-\operatorname{div}_{v}\left(D_{v} u m\right)=0, & \text { in } \mathbb{R}^{2 N} \times(0, T), \\
(\text { iii }) & m(x, v, 0)=m_{0}(x, v), u(x, v, T)=G[m(T)](x, v), & \text { on } \mathbb{R}^{2 N},
\end{array}\right.
$$

which corresponds to $H\left(x, v, p_{v}\right)=\frac{1}{2}\left|p_{v}\right|^{2}-\frac{1}{2}|v|^{2}-l(x, v)$.

Let $\mathcal{P}_{1}$ and $\mathcal{P}_{2}$ denote the spaces of Borel probability measures on $\mathbb{R}^{2 N}$ with respectively finite first and second order moments, endowed with the Monge-Kantorovich distances $\mathbf{d}_{1}$, respectively $\mathbf{d}_{2}$.

Let $C^{2}\left(\mathbb{R}^{2 N}\right)$ denote the space of twice differentiable functions with continuous and bounded derivatives up to order two. It is endowed with the norm

$\|f\|_{C^{2}}:=\sup _{(x, v) \in \mathbb{R}^{2 N}}\left[|f(x, v)|+|D f(x, v)|+\left|D^{2} f(x, v)\right|\right]$.

Hereafter, we shall make the following hypotheses:

\section{Assumptions ( $\mathbf{H})$}

(H1) The functions $F$ and $G$ are real-valued continuous functions defined on $\mathcal{P}_{1} \times \mathbb{R}^{2 N}$

(H2) The function $l$ is a real-valued $C^{2}$ function defined on $\mathbb{R}^{2 N}$

(H3) The map $m \mapsto F[m](\cdot, \cdot)$ is Lipschitz continuous from $\mathcal{P}_{1}$ to $C^{2}\left(\mathbb{R}^{2 N}\right)$; moreover, there exists $C>0$ such that $C \geqslant\|l\|_{C^{2}}$ and

$$
\|F[m](\cdot, \cdot)\|_{C^{2}}+\|G[m](\cdot, \cdot)\|_{C^{2}} \leqslant C, \quad \forall m \in \mathcal{P}_{1}
$$

(H4) the initial distribution $m_{0}$, defined on $\mathbb{R}^{2 N}$, has a compactly supported density (still named $m_{0}$, with a slight abuse of notation) $m_{0} \in C^{0, \delta}\left(\mathbb{R}^{2 N}\right)$ for some $\delta \in(0,1)$.

Definition 2.1. The pair $(u, m)$ is a solution of system (2.1) if:

1) $u \in W_{\text {loc }}^{1, \infty}\left(\mathbb{R}^{2 N} \times[0, T]\right), m \in C\left([0, T] ; \mathcal{P}_{1}\left(\mathbb{R}^{2 N}\right)\right)$ and for all $t \in[0, T], m(t)$ is absolutely continuous with respect to Lebesgue measure on $\mathbb{R}^{2 N}$. Let $m(\cdot, \cdot, t)$ denote the density of $m(t)$. The function $(x, v, t) \mapsto m(x, v, t)$ is bounded.

2) equation (2.1)-(i) is satisfied by $u$ in the viscosity sense

3) equation (2.1)-(ii) is satisfied by $m$ in the sense of distributions.

We can now state the main result of this paper:

Theorem 2.1. Under the assumptions $(\mathrm{H})$ :

1. System (2.1) has a solution $(u, m)$ in the sense of Definition 2.1,

2. $m$ is the image of $m_{0}$ by the flow

$$
\begin{cases}x^{\prime}(s)=v(s) & x(0)=x \\ v^{\prime}(s)=-D_{v} u(x(s), v(s), s), & v(0)=v .\end{cases}
$$

Proposition 2.1. Under the additional assumptions

$$
\int_{\mathbb{R}^{2 N}}\left(F\left[m_{1}\right]-F\left[m_{2}\right]\right) d\left(m_{1}-m_{2}\right)>0 \quad \text { and } \int_{\mathbb{R}^{2 N}}\left(G\left[m_{1}\right]-G\left[m_{2}\right]\right) d\left(m_{1}-m_{2}\right) \geqslant 0
$$

for every $m_{1}, m_{2} \in \mathcal{P}_{1}\left(\mathbb{R}^{2 N}\right), m_{1} \neq m_{2}$, the solution found in Theorem 2.1 is unique. 


\section{The optimal control problem}

In this section, we tackle the optimal control problem related to equation (2.1)-(i) with a fixed $\bar{m} \in C\left([0, T] ; \mathcal{P}_{1}\left(\mathbb{R}^{2 N}\right)\right)$. To simplify the notation, we introduce the functions

$$
\ell(x, v, t):=l(x, v)+F[\bar{m}(t)](x, v) \quad \text { and } \quad g(x, v):=G[\bar{m}(T)](x, v),
$$

which, from the set assumptions $(\mathrm{H})$, satisfy

$$
\|\ell(\cdot, \cdot, t)\|_{C^{2}},\|\ell(x, v, \cdot)\|_{C},\|g\|_{C^{2}} \leqslant C \quad \forall t \in[0, T],(x, v) \in \mathbb{R}^{2 N} .
$$

With the new notation, the optimal control problem to be solved by a representative agent whose state at time $t$ is $(x, v)$ is to find the control law $\alpha$ in order to minimize

$$
J_{t}(\xi, \eta, \alpha)=\int_{t}^{T}\left[\frac{|\alpha|^{2}}{2}+\frac{|\eta|^{2}}{2}+\ell(\xi(s), \eta(s), s)\right] d s+g(\xi(T), \eta(T))
$$

by following the trajectory (1.3). Then the Cauchy problem given by (2.1)-(i) and its terminal condition becomes

$$
\begin{cases}-\partial_{t} u-v \cdot D_{x} u+\frac{1}{2}\left|D_{v} u\right|^{2}-\frac{1}{2}|v|^{2}-\ell(x, v, t)=0 & \text { in } \mathbb{R}^{2 N} \times(0, T), \\ u(x, v, T)=g(x, v) & \text { on } \mathbb{R}^{2 N} .\end{cases}
$$

From (3.3), it is obvious that the control $\alpha$ must be chosen in $L^{2}\left(t, T ; \mathbb{R}^{N}\right)$. Therefore, we can introduce the value function as follows:

Definition 3.1. The value function for the cost $J_{t}$ defined in (3.3) with dynamics (1.3) is

$$
u(x, v, t):=\inf \left\{J_{t}(\xi, \eta, \alpha):(\xi, \eta, \alpha) \in \mathcal{A}(x, v, t)\right\}
$$

where

$$
\mathcal{A}(x, v, t)=\left\{\begin{array}{l|l}
(\xi, \eta, \alpha): & \begin{array}{l}
(\xi, \eta) \in A C\left([t, T] ; \mathbb{R}^{2 N}\right), \alpha \in L^{2}\left(t, T ; \mathbb{R}^{N}\right), \\
(\xi, \eta, \alpha) \text { satisfy }(1.3) \text { and } \xi(t)=x, \eta(t)=v
\end{array}
\end{array}\right\} .
$$

Lemma 3.1. i) (Existence of an optimal control.) For every $(x, v, t) \in \mathbb{R}^{N} \times \mathbb{R}^{N} \times$ $(0, T)$, there exists an optimal control $\alpha^{*}$ for $u(x, v, t)$.

ii) (Concatenation.) Let $\left(\xi^{*}, \eta^{*}\right)$ be an optimal trajectory for $u(x, v, t)$ corresponding to the control law $\alpha^{*}$. For $r \in(t, T)$, let $\left(\tilde{\xi}^{*}, \tilde{\eta}^{*}\right)$ be an optimal trajectory for $u\left(\xi^{*}(r), \eta^{*}(r), r\right)$ with control $\tilde{\alpha}^{*}$. Then the concatenation of $\alpha^{*}$ and $\tilde{\alpha}^{*}$ at time $r$ is optimal for $u(x, v, t)$ and, moreover,

$$
u(x, v, t)=u\left(\xi^{*}(r), \eta^{*}(r), r\right)+\int_{t}^{r}\left[\frac{\left|\alpha^{*}\right|^{2}}{2}+\frac{\left|\eta^{*}\right|^{2}}{2}+\ell\left(\xi^{*}(s), \eta^{*}(s), s\right)\right] d s .
$$

iii) Under the same assumption as in point (ii), the control $\alpha_{\mid[r, T]}^{*}$ is optimal for $u\left(\xi^{*}(r), \eta^{*}(r), r\right)$.

iv) (Dynamic Programming Principle.) The Dynamic Programming Principle holds, namely

$$
u(x, v, t)=\min _{(\xi, \eta, \alpha) \in \mathcal{A}(x, v, t)}\left\{u(\xi(r), \eta(r), r)+\int_{t}^{r} \frac{|\alpha(s)|^{2}}{2}+\frac{|\eta(s)|^{2}}{2}+\ell(\xi(s), \eta(s), s) d s\right\} .
$$


Proof. (i): let $\left\{\alpha_{n}\right\}_{n}$ be a sequence of minimizing control laws and $\left(\xi_{n}, \eta_{n}\right)$ be the solution of (1.3) corresponding to $\alpha_{n}$. Then, the boundedness of $\ell$ and the definition of $J_{t}$ ensure that $\left\|\alpha_{n}\right\|_{L^{2}\left(t, T ; \mathbb{R}^{N}\right)}$ are uniformly bounded. Then, possibly after extracting a subsequence, $\alpha_{n} \rightarrow \alpha^{*}$ in $L^{2}\left(t, T ; \mathbb{R}^{N}\right), \eta_{n} \rightarrow \eta^{*}$ in $C\left([t, T] ; \mathbb{R}^{N}\right)$ and $\xi_{n} \rightarrow \xi^{*}$ in $C^{1}\left([t, T] ; \mathbb{R}^{N}\right)$. The lower semi-continuity of $J_{t}$ yields that $\alpha^{*}$ is optimal.

Points (ii), (iii) and (iv) are obtained by arguing exactly as in [29, Proposition 5.1] (points (1), (2) and (4) respectively), see also [12].

Lemma 3.2. The value function $u$ has the following properties:

1. (Lipschitz continuity in $x$ and local Lipschitz continuity in $v$ ) there exists a positive constant $C$, depending only on the constants in assumptions $(\mathrm{H})$, such that

$$
\begin{aligned}
& \left|u(x, v, t)-u\left(x^{\prime}, v, t\right)\right| \leqslant C\left|x-x^{\prime}\right| \\
& \left|u(x, v, t)-u\left(x, v^{\prime}, t\right)\right| \leqslant C\left(1+|v|+\left|v^{\prime}\right|\right)\left|v-v^{\prime}\right|
\end{aligned}
$$

for every $x, x^{\prime}, v, v^{\prime} \in \mathbb{R}^{N}, t \in[0, T]$.

2. (Local Lipschitz continuity in $t$ ) there exists a positive constant $C$, depending only on the constants in assumptions $(\mathrm{H})$, such that

$$
\left|u(x, v, t)-u\left(x, v, t^{\prime}\right)\right| \leqslant C\left(1+|v|^{2}\right)\left|t-t^{\prime}\right| \quad \forall x, v \in \mathbb{R}^{N}, t, t^{\prime} \in[0, T] .
$$

Proof. 1. Fix $t \in[0, T)$. Let $\alpha$ be an optimal control law for $u(x, v, t)$ i.e.,

$$
u(x, v, t)=\int_{t}^{T} \frac{1}{2}|\alpha(s)|^{2}+\frac{1}{2}|v(s)|^{2}+\ell(x(s), v(s), s) d s+g(x(T), v(T)),
$$

where $(x(\cdot), v(\cdot))$ obeys to the dynamics $(1.3)$.

We consider the path $(y(\cdot), w(\cdot))$ starting from $(y, w)$, with control $\alpha(\cdot)$. Hence, we obtain

$$
\begin{aligned}
& y(s)=y+w(s-t)+\int_{t}^{s} \int_{t}^{\theta} \alpha(\tau) d \tau d \theta=y-x+x(s)+(w-v)(s-t), \\
& w(s)=w+\int_{t}^{s} \alpha(\tau) d \tau=w-v+v(s) .
\end{aligned}
$$

Note that

$$
v(s)-w(s)=v-w, x(s)-y(s)=x-y+(v-w)(s-t) .
$$

The definition of the value function (3.5) and relation (3.7) imply

$$
\begin{aligned}
u(y, w, t) \leqslant & \int_{t}^{T} \frac{1}{2}|\alpha(s)|^{2}+\frac{1}{2}|w(s)|^{2}+\ell(y(s), w(s), s) d s+g(y(T), w(T)) \\
\leqslant & u(x, v, t)-\int_{t}^{T} \frac{1}{2}|v(s)|^{2}-\ell(x(s), v(s), s) d s-g(x(T), v(T)) \\
& \quad+\int_{t}^{T} \frac{1}{2}|w(s)|^{2}+\ell(y(s), w(s), s) d s+g(y(T), w(T)) \\
\leqslant & u(x, v, t)+\int_{t}^{T} L_{\ell}(|x(s)-y(s)|+|v(s)-w(s)|) d s \\
& \quad+L_{g}(|x(T)-y(T)|+|v(T)-w(T)|)+\int_{t}^{T} \frac{1}{2}\left(|w(s)|^{2}-|v(s)|^{2}\right) d s,
\end{aligned}
$$


where $L_{\ell}$ and $L_{g}$ denote respectively the Lipschitz constants of $\ell$ and $g$ w.r.t. $(x, v)$. Hence, by (3.8),

$$
\begin{aligned}
& \int_{t}^{T} \frac{1}{2}\left(|w(s)|^{2}-|v(s)|^{2}\right) d s=\int_{t}^{T} \frac{1}{2}|w-v| \cdot|w(s)+v(s)| d s \leqslant \\
& |w-v| \int_{t}^{T}\left|w+v+2 \int_{t}^{s} \alpha(\tau) d \tau\right| d s \leqslant C|w-v|(|w|+|v|+1),
\end{aligned}
$$

where the last inequality comes from (3.33) of Corollary 3.1 below. Hence we obtain

$$
u(y, w, t) \leqslant u(x, v, t)+C|x-y|+K(v, w)|v-w|,
$$

where $K(v, w)=C(|w|+|v|+1)$. Reverting the roles of $(x, v)$ and $(y, w)$, we get the first result.

2. We fix $(x, v)$. From the concatenation property of optimal trajectories established in Lemma 3.1, if $\alpha$ is optimal for $u(x, v, t)$ and $(x(s), v(s))$ is the associated optimal trajectory, then

$$
u(x, v, t)=u(x(s), v(s), s)+\int_{t}^{s} \frac{1}{2}|\alpha(r)|^{2}+\frac{1}{2}|v(r)|^{2}+\ell(x(r), v(r), r) d r
$$

for any $s \in[t, T]$. Then

$$
\begin{aligned}
& |u(x, v, t)-u(x, v, s)| \leqslant|u(x, v, t)-u(x(s), v(s), s)|+|u(x(s), v(s), s)-u(x, v, s)| \\
& \leqslant \int_{t}^{s} \frac{1}{2}|\alpha(r)|^{2}+\frac{1}{2}|v(r)|^{2}+|\ell(x(r), v(r), r)| d r+L|x(s)-x|+L(v)|v(s)-v|,
\end{aligned}
$$

where the last two terms come from the Lipschitz continuity of $u$ w.r.t. $(x, v)$ : $L$ is the Lipschitz constant of $u$ with respect to $x$ and $L(v)$ is a local Lipschitz constant of $u$ with respect to $v$.

From (1.3) and the bound (3.33) in Corollary 3.1 below, we get the bounds for $x(s)$, $v(s)$ and $\alpha$, i.e. $|v(s)-v|+|x(s)-x| \leqslant C(1+|v|)|s-t|$, hence

$$
|u(x, v, t)-u(x, v, s)| \leqslant C\left(1+|v|^{2}\right)|s-t|
$$

which ends the proof.

Proposition 3.1. The value function defined in (3.5) is the unique viscosity solution to (3.4) with an at most quadratic growth in $(x, v)$. Moreover, there exists a positive constant $C$ such that

$$
-C \leqslant u(x, v, t) \leqslant C\left(1+|v|^{2}\right) \quad \forall(x, v, t) \in \mathbb{R}^{N} \times \mathbb{R}^{N} \times[0, T] .
$$

Proof. Let us first establish that the value function fulfills (3.11) and solves (3.4) in the viscosity sense. Actually, taking $\alpha \equiv 0$ in (1.3), we get $\eta(s)=v$ and $\xi(s)=x+v(s-t)$; then, thanks to the boundedness of $\ell$ in (3.2), the value function verifies (3.11). Moreover, by Lemma 3.2, it is also continuous; hence, using the DPP in Lemma 3.1-(iv), it is also a solution to $(3.4)$.

The uniqueness part of the statement is an immediate consequence of the comparison principle stated in [19, Theorem 2.1]. 
The following lemma deals with the semi-concavity of $u(x, v, t)$ w.r.t. $(x, v)$ :

Lemma 3.3. Under Hypothesis $(\mathrm{H}), u(x, v, t)$ is semi-concave w.r.t. $(x, v)$ with a linear modulus of semi-concavity, which depends only on the constants in assumptions $(\mathrm{H})$.

Proof. For any $(x, v),(y, w)$ and $\lambda \in[0,1]$, consider $x_{\lambda}:=\lambda x+(1-\lambda) y, v_{\lambda}:=\lambda v+(1-\lambda) w$.

Let $\alpha$ be an optimal control for $u\left(x_{\lambda}, v_{\lambda}, t\right)$; hence, the associated trajectory is

$$
x_{\lambda}(s)=x_{\lambda}+v_{\lambda}(s-t)+\int_{t}^{s} \int_{t}^{\theta} \alpha(\tau) d \tau d \theta, v_{\lambda}(s)=v_{\lambda}+\int_{t}^{s} \alpha(\tau) d \tau
$$

and

$$
u\left(x_{\lambda}, v_{\lambda}, t\right)=\int_{t}^{T} \frac{1}{2}|\alpha(s)|^{2}+\frac{1}{2}\left|v_{\lambda}(s)\right|^{2}+\ell\left(x_{\lambda}(s), v_{\lambda}(s), s\right) d s+g\left(x_{\lambda}(T), v_{\lambda}(T)\right) .
$$

Let $(x(s), v(s))$ be the trajectory starting at $(x, v)$ at time $t$ with control $\alpha$ and $(y(s), w(s))$ the trajectory starting at $(y, w)$ at time $t$ still with control $\alpha$.

We have to estimate

$$
\begin{aligned}
& \lambda u(x, v, t)+(1-\lambda) u(y, w, t)-u\left(x_{\lambda}, v_{\lambda}, t\right) \\
\leqslant & \int_{t}^{T} \frac{1}{2} \lambda|v(s)|^{2}+(1-\lambda) \frac{1}{2}|w(s)|^{2}-\frac{1}{2}\left|v_{\lambda}(s)\right|^{2} d s \\
& +\int_{t}^{T} \lambda \ell(x(s), v(s), s)+(1-\lambda) \ell(y(s), w(s), s)-\ell\left(x_{\lambda}(s), v_{\lambda}(s), s\right) d s \\
& +\lambda g(x(T), v(T))+(1-\lambda) g(y(T), w(T))-g\left(x_{\lambda}(T), v_{\lambda}(T)\right) .
\end{aligned}
$$

Since

$$
v(s)=v+\int_{t}^{s} \alpha(\tau) d \tau, w(s)=w+\int_{t}^{s} \alpha(\tau) d \tau, v_{\lambda}(s)=\lambda v+(1-\lambda) w+\int_{t}^{s} \alpha(\tau) d \tau
$$

we get

$$
\begin{aligned}
& \lambda \frac{1}{2}|v(s)|^{2}+(1-\lambda) \frac{1}{2}|w(s)|^{2}-\frac{1}{2}\left|v_{\lambda}(s)\right|^{2} \\
= & (\lambda v+(1-\lambda) w-\lambda v-(1-\lambda) w) \int_{t}^{s} \alpha(\tau) d \tau+\lambda \frac{|v|^{2}}{2}+(1-\lambda) \frac{|w|^{2}}{2}-\frac{1}{2}|\lambda v+(1-\lambda) w|^{2} \\
= & \frac{1}{2} \lambda(1-\lambda)|v|^{2}+\frac{1}{2} \lambda(1-\lambda)|w|^{2}-\lambda(1-\lambda) v \cdot w=\frac{1}{2} \lambda(1-\lambda)|v-w|^{2} .
\end{aligned}
$$

Hence

$$
\int_{t}^{T} \frac{1}{2} \lambda|v(s)|^{2}+(1-\lambda) \frac{1}{2}|w(s)|^{2}-\frac{1}{2}\left|v_{\lambda}(s)\right|^{2} d s=\frac{1}{2} \lambda(1-\lambda)|v-w|^{2}(T-t) .
$$

Now, we have to estimate the terms $\lambda \ell(x(s), v(s), s)+(1-\lambda) \ell(y(s), w(s), s)-\ell\left(x_{\lambda}(s), v_{\lambda}(s), s\right)$ and $\lambda g(x(T), v(T))+(1-\lambda) g(y(T), w(T))-g\left(x_{\lambda}(T), v_{\lambda}(T)\right)$. We write the algebra for the second term, since the treatment of the first term is similar. The Taylor expansion of $g$ centered at $\left(x_{\lambda}(T), v_{\lambda}(T)\right)$ gives

$$
g(x(T), v(T))=g\left(x_{\lambda}(T), v_{\lambda}(T)\right)+D g\left(x_{\lambda}(T), v_{\lambda}(T)\right)\left(x(T)-x_{\lambda}(T), v(T)-v_{\lambda}(T)\right)+R_{1},
$$


where $R_{1}$ is the remaining term in the expansion, namely

$$
R_{1}=\frac{1}{2}\left(x(T)-x_{\lambda}(T), v(T)-v_{\lambda}(T)\right) D^{2} g\left(\xi_{1}, \eta_{1}\right)\left(x(T)-x_{\lambda}(T), v(T)-v_{\lambda}(T)\right)^{T},
$$

for suitable $\xi_{1}, \eta_{1}$.

From (3.12) and (3.13), we get

$$
\begin{aligned}
x(s)-x_{\lambda}(s) & =(1-\lambda)((x-y)+(v-w)(s-t)), \\
v(s)-v_{\lambda}(s) & =(1-\lambda)(v-w), \\
y(s)-x_{\lambda}(s) & =\lambda((y-x)+(w-v)(s-t)), \\
w(s)-v_{\lambda}(s) & =\lambda(w-v),
\end{aligned}
$$

hence the error term can be written as

$$
R_{1}=\frac{1}{2}(1-\lambda)^{2}(x-y+(v-w)(T-t), v-w) D^{2} g\left(\xi_{1}, \eta_{1}\right)(x-y+(v-w)(T-t), v-w)^{T}
$$

Similarly

$g(y(T), w(T))=g\left(x_{\lambda}(T), v_{\lambda}(T)\right)+D g\left(x_{\lambda}(T), v_{\lambda}(T)\right)\left(y(T)-x_{\lambda}(T), w(T)-v_{\lambda}(T)\right)+R_{2}$,

where

$$
\begin{aligned}
R_{2} & =\frac{1}{2}\left(y(T)-x_{\lambda}(T), w(T)-v_{\lambda}(T)\right) D^{2} g\left(\xi_{2}, \eta_{2}\right)\left(y(T)-x_{\lambda}(T), w(T)-v_{\lambda}(T)\right)^{T} \\
& =\frac{1}{2} \lambda^{2}(y-x+(w-v)(T-t), w-v) D^{2} g\left(\xi_{2}, \eta_{2}\right)(y-x+(w-v)(T-t), w-v)^{T} .
\end{aligned}
$$

At this point, taking into account that from (3.18),

$$
\begin{aligned}
& \lambda D g\left(x_{\lambda}(T), v_{\lambda}(T)\right)\left(x(T)-x_{\lambda}(T), v(T)-v_{\lambda}(T)\right) \\
& +(1-\lambda) D g\left(x_{\lambda}(T), v_{\lambda}(T)\right)\left(y(T)-x_{\lambda}(T), w(T)-v_{\lambda}(T)\right) \\
= & D g\left(x_{\lambda}(T), v_{\lambda}(T)\right)\left(\lambda\left(x(T)-x_{\lambda}(T)\right)\right. \\
& \left.+(1-\lambda)\left(y(T)-x_{\lambda}(T)\right), \lambda\left(v(T)-v_{\lambda}(T)\right)+(1-\lambda)\left(w(T)-v_{\lambda}(T)\right)\right) \\
= & 0
\end{aligned}
$$

we obtain that

$$
\begin{aligned}
& \lambda g(x(T), v(T))+(1-\lambda) g(y(T), w(T))-g\left(x_{\lambda}(T), v_{\lambda}(T)\right) \\
= & \lambda R_{1}+(1-\lambda) R_{2} \\
\leqslant & (1-\lambda) \lambda C_{T}\left\|D^{2} g\right\|_{\infty}\left(|x-y|^{2}+|v-w|^{2}\right) .
\end{aligned}
$$

Hence from (3.15), (3.20), (3.21) we get

$$
\begin{aligned}
& \lambda u(x, v, t)+(1-\lambda) u(y, w, t)-u\left(x_{\lambda}, v_{\lambda}, t\right) \\
\leqslant & \frac{\lambda(1-\lambda)}{2}|v-w|^{2}(T-t)+C_{T}(1-\lambda) \lambda\left(\left\|D^{2} g\right\|_{\infty}+\left\|D^{2} \ell\right\|_{\infty}\right)\left(|x-y|^{2}+|v-w|^{2}\right) .
\end{aligned}
$$

We obtain that $u$ is semi-concave in $(x, v)$ with a linear modulus of semi-concavity. tions:

Pontryagin's maximum principle yields the following necessary optimality condi- 
Proposition 3.2 (Necessary conditions for optimality). Let $\left(x^{*}, v^{*}, \alpha^{*}\right)$ be optimal for $u(x, v, t)$ in (3.5). There exists an arc $p=\left(p_{x}, p_{v}\right) \in A C\left([t, T] ; \mathbb{R}^{N} \times \mathbb{R}^{N}\right)$, hereafter called the costate, such that

1. $\left(\alpha^{*}, x^{*}, v^{*}, p\right)$ satisfies the adjoint equations: for a.e. $s \in[t, T]$,

$$
\begin{aligned}
& p_{x}^{\prime}=D_{x} \ell\left(x^{*}, v^{*}, s\right), \\
& p_{v}^{\prime}=-p_{x}+v^{*}+D_{v} \ell\left(x^{*}, v^{*}, s\right),
\end{aligned}
$$

the transversality condition

$$
p(T)=-D g\left(x^{*}(T), v^{*}(T)\right),
$$

together with the maximum condition: for almost all $s \in[t, T]$,

$$
\max _{\alpha} p_{x} \cdot v^{*}+p_{v} \cdot \alpha-\frac{|\alpha|^{2}}{2}-\frac{\left|v^{*}\right|^{2}}{2}=p_{x} \cdot v^{*}+p_{v} \cdot \alpha^{*}-\frac{\left|\alpha^{*}\right|^{2}}{2}-\frac{\left|v^{*}\right|^{2}}{2}
$$

2. The optimal control $\alpha^{*}$ is given by

$$
\alpha^{*}=p_{v} \text {, a.e in }[t, T] .
$$

3. The triple $\left(x^{*}, v^{*}, p\right)$ satisfies the system of differential equations: for a.e. $s \in[t, T]$

$$
\begin{aligned}
& x^{\prime}=v, \\
& v^{\prime}=p_{v}, \\
& p_{x}^{\prime}=D_{x} \ell(x, v, s), \\
& p_{v}^{\prime}=-p_{x}+v+D_{v} \ell(x, v, s),
\end{aligned}
$$

with the mixed boundary conditions $x^{*}(t)=x, v^{*}(t)=v, p(T)=-D g\left(x^{*}(T), v^{*}(T)\right)$.

Proof. 1. Hypothesis (3.2) ensures that our control problem satisfies the assumption [18, Hypothesis 22.16], so we can invoke [18, Theorem 22.17] on the maximum principle for problems with unbounded control. Moreover, since there is no constraint on the state variable at $T$, the same arguments as in [18, Corollary 22.3] ensure that the necessary conditions hold in normal form.

2. The maximum condition (3.25) implies that

$$
D_{\alpha}\left(p_{x} \cdot v^{*}+p_{v} \cdot \alpha-\frac{|\alpha|^{2}}{2}-\frac{\left|v^{*}\right|^{2}}{2}-f\left(x^{*}, v^{*}\right)\right)_{\alpha=\alpha^{*}}=0 \quad \text { for a.e. } s \in[t, T]
$$

from which we get (3.26).

3. Conditions (3.27) - (3.28) follow directly from (1.3) and (3.26). Conditions (3.29) and (3.30) coincide with (3.22), (3.23).

Corollary 3.1 (Feedback control and regularity). Let $\left(x^{*}, v^{*}, \alpha^{*}\right)$ be optimal for $u(x, v, t)$ and $p=\left(p_{x}, p_{v}\right)$ be the related costate as in Proposition 3.2. Then:

1. The costate $p$ is uniquely expressed in terms of $x^{*}, v^{*}$ for every $s \in[t, T]$ by

$$
\left\{\begin{array}{l}
p_{x}(s)=-D_{x} g\left(x^{*}(T), v^{*}(T)\right)-\int_{s}^{T} D_{x} \ell\left(x^{*}(\tau), v^{*}(\tau), \tau\right) d \tau \\
p_{v}(s)=-D_{v} g\left(x^{*}(T), v^{*}(T)\right)-\int_{s}^{T} D_{v} \ell\left(x^{*}(\tau), v^{*}(\tau), \tau\right)+v^{*}(\tau)-p_{x}(\tau) d \tau .
\end{array}\right.
$$


2. The optimal control $\alpha^{*}$ is a feedback control (i.e., a function of $x^{*}, v^{*}$ ), uniquely expressed in terms of $x^{*}, v^{*}$ for a.e. $s \in[t, T]$ by

$$
\alpha^{*}(s)=p_{v}(s) \text {. }
$$

3. The optimal trajectory $\left(x^{*}, v^{*}\right)$ and the optimal control $\alpha^{*}$ are of class $C^{1}$. In particular the equalities (3.26) - (3.32) do hold for every $s \in[t, T]$. Moreover

$$
\begin{aligned}
\left\|v^{*}\right\|_{C^{1}}+\left\|\alpha^{*}\right\|_{C^{1}} & \leqslant C(1+|v|), \\
\left\|x^{*}\right\|_{C^{1}} & \leqslant|x|+C(1+|v|) .
\end{aligned}
$$

4. Assume that, for some $k \in \mathbb{N}, D_{x} \ell(x, v, s), D_{v} \ell(x, v, s)$ are of class $C^{k}$. Then $\left(x^{*}, v^{*}\right), p$ and $\alpha^{*}$ are of class $C^{k+1}$.

Proof. Point 1 is obtained integrating (3.29)-(3.30) and taking into account the final time condition $p(T)=-D g\left(x^{*}(T), v^{*}(T)\right)$. Point 2 follows from (3.26).

Proof of point 3. Since $x^{*}, v^{*}$ are continuous by the definition of admissible trajectories in (3.6), the continuity of $\alpha^{*}$ follows from (3.31) and (3.32). Then (1.3) implies $v^{*} \in C^{1}$ and also $x^{*} \in C^{1}$. Relations (3.31), (3.32) (and the regularity of $\ell$ ) imply, respectively, that $p$ and $\alpha^{*}$ are of class $C^{1}$. By (3.28), we get that $v^{*}$ is $C^{2}$. Let us now prove the bounds (3.33). To this end, we observe that equations (3.28) and (3.30) entail

$$
\left(v^{*}\right)^{\prime \prime}(\tau)-v^{*}(\tau)=-p_{x}(\tau)+D_{v} \ell\left(x^{*}(\tau), v^{*}(\tau), \tau\right)
$$

where, by (3.31) and (H2), the right hand side is bounded uniformly in $x$ and $v, \tau \in[t, T]$. Moreover,

- $\left(v^{*}\right)(t)=v$

- by (3.28), (3.24) and the regularity of $g,\left(v^{*}\right)^{\prime}(T)$ is bounded uniformly in $x$ and $v$.

Hence, using the method of variation of constants for the above ordinary differential equation with assigned the values of $v^{*}(t)$ and of $\left(v^{*}\right)^{\prime}(T)$, we get the estimate for $v^{*}$ and for $\alpha^{*}$. Integrating $v^{*}$, we get the estimate for $x^{*}$.

Proof of point 4. The relations (3.31) and the $C^{1}$-regularity of $x^{*}, v^{*}$ and $p$ imply that, actually, $p \in C^{2}$. Therefore, (3.32) gives the $C^{2}$-regularity of $\alpha^{*}$ and, finally, (2.2) yields the $C^{2}$-regularity of $x^{*}, v^{*}$. Further regularity of $x^{*}, v^{*}, \alpha^{*}$ and $p$ follows by a standard bootstrap inductive argument.

Remark 3.1. Taking advantage of Corollary 3.1-(3), we will always consider the representation of the optimal control $\alpha^{*}$ which belongs to $C^{1}$.

Corollary 3.2 that follows implies that the optimal trajectories for $u(x, v, t)$ do not bifurcate at any time $r>t$.

Corollary 3.2. Under Hypothesis (3.2), let $\left(x^{*}, v^{*}\right)$ be an optimal trajectory for $u(x, v, t)$. For every $t<r<T$, there are no other optimal trajectories for $u\left(x^{*}(r), v^{*}(r), r\right)$ other than $\left(x^{*}, v^{*}\right)$ restricted to $[r, T]$. 
Proof. 1. Let $r \in(t, T)$ and $\left(y^{*}, w^{*}\right)$ be an optimal trajectory for $u\left(x^{*}(r), v^{*}(r), r\right)$. Lemma 3.1 ensures that $\left(z^{*}, \nu^{*}\right)$, the concatenation of $\left(x^{*}, v^{*}\right)$ with $\left(y^{*}, w^{*}\right)$ at $r$ is an optimal trajectory for $u(x, v, t)$. Let $p:=\left(p_{x}, p_{v}\right), q:=\left(q_{x}, q_{v}\right)$ be the costates corresponding respectively to $\left(x^{*}, v^{*}\right)$ and to $\left(z^{*}, \nu^{*}\right)$. Both $\left(x^{*}, v^{*}, p\right)$ and $\left(z^{*}, \nu^{*}, q\right)$ satisfy $(3.27)-$ (3.30) on $[t, T]$. Now, Corollary 3.1 shows that $\left(x^{*}, v^{*}\right)$ and $\left(z^{*}, \nu^{*}\right)$ are of class $C^{1}$. Since $x^{*}=z^{*}, v^{*}=\nu^{*}$ on $[t, r]$, we choose $\tau$ such that $t<\tau<r$. From (3.28), we get

$$
p_{v}(\tau)=q_{v}(\tau)
$$

Moreover, from (3.28) and (3.30), we also get that

$$
p_{x}(\tau)=q_{x}(\tau) .
$$

Therefore, both $\left(x^{*}, v^{*}, p\right)$ and $\left(z^{*}, \nu^{*}, q\right)$ are solutions to the same Cauchy problem on $[t, T]$ with the first order differential system (3.27)-(3.30) and Cauchy data at $\tau$. The regularity assumptions on $\ell, g$ and Cauchy-Lipschitz Theorem guarantee the uniqueness of the solution. Thus $x^{*}=z^{*}, v^{*}=\nu^{*}$ on $[\tau, T]$, from which we obtain the desired identities $x^{*}=y^{*}$ and $v^{*}=w^{*}$ on $[r, T]$.

Definition 3.2. For any $(x, v, t) \in \mathbb{R}^{2 N} \times[0, T]$, let $\mathcal{U}(x, v, t)$ denote the set of optimal controls for the value function $u(x, v, t)$ defined in (3.5).

Remark 3.2. Lemma 3.1-(i) and Remark 3.1 ensure that $\varnothing \neq \mathcal{U}(x, v, t) \subset C^{1}\left([t, T] ; \mathbb{R}^{N}\right)$.

Lemma 3.4. The following properties hold:

1. The function $u(x, \cdot, t)$ is differentiable at $v$ if and only if the set $\{\alpha(t): \alpha \in \mathcal{U}(x, v, t)\}$ is a singleton. Moreover $D_{v} u(x, v, t)=-\alpha(t)$.

2. In particular, if $\mathcal{U}(x, v, t)$ is a singleton, then, calling $(x(s), v(s))$ the optimal trajectory associated to the singleton $\mathcal{U}(x, v, t), D_{v} u(x(s), v(s), s)$ exists for any $s \in[t, T]$.

3. If $u(\cdot, \cdot, t)$ is differentiable at $(x, v)$, then $\mathcal{U}(x, v, t)$ is a singleton.

Proof. 1. We prove that if $D_{v} u(x, v, t)$ exists, then all $\alpha(\cdot) \in \mathcal{U}(x, v, t)$ take the same value $\alpha(t)$ at $t$ and $D_{v} u(x, v, t)=-\alpha(t)$. If $\alpha(\cdot) \in \mathcal{U}(x, v, t)$, calling $(x(\cdot), v(\cdot))$ the corresponding optimal trajectory, then

$$
u(x, v, t)=\int_{t}^{T} \frac{1}{2}|\alpha(s)|^{2}+\frac{1}{2}|v(s)|^{2}+\ell(x(s), v(s), s) d s+g(x(T), v(T)),
$$

and $(x(\cdot), v(\cdot))$ and $\alpha(\cdot)$ satisfy the necessary conditions for optimality proved in Proposition 3.2. Take $h=\left(h_{1}, h_{2}\right) \in \mathbb{R}^{2 N}$ and consider the solution $(y(\cdot), w(\cdot))$ of (1.3) with initial condition $(y(t), w(t))=\left(x+h_{1}, v+h_{2}\right)$ and control $\alpha$, namely

$$
\begin{aligned}
& y(s)=x+h_{1}+\left(v+h_{2}\right)(s-t)+\int_{t}^{s} \int_{t}^{\theta} \alpha(\tau) d \tau d \theta=x(s)+h_{1}+h_{2}(s-t), \\
& w(s)=v+h_{2}+\int_{t}^{s} \alpha(\tau) d \tau=v(s)+h_{2} .
\end{aligned}
$$


Hence,

$$
\begin{aligned}
& u\left(x+h_{1}, v+h_{2}, t\right)-u(x, v, t) \\
\leqslant & \int_{t}^{T} \frac{1}{2}|w(s)|^{2}-\frac{1}{2}|v(s)|^{2}+\ell(y(s), w(s), s)-\ell(x(s), v(s), s) d s \\
& +g(y(T), w(T))-g(x(T), v(T)) \\
= & \int_{t}^{T} \frac{1}{2}\left|v(s)+h_{2}\right|^{2}-\frac{1}{2}|v(s)|^{2}+\ell\left(x(s)+h_{1}+h_{2}(s-t), v(s)+h_{2}, s\right)-\ell(x(s), v(s), s) d s \\
& +g\left(x(T)+h_{1}+h_{2}(T-t), v(T)+h_{2}\right)-g(x(T), v(T)) \\
= & \int_{t}^{T} \frac{1}{2} h_{2}^{2}+h_{2} \cdot v(s)+\ell\left(x(s)+h_{1}+h_{2}(s-t), v(s)+h_{2}, s\right)-\ell(x(s), v(s), s) d s \\
& +g\left(x(T)+h_{1}+h_{2}(T-t), v(T)+h_{2}\right)-g(x(T), v(T)) .
\end{aligned}
$$

The arbitrariness of the sign of the components of $\left(h_{1}, h_{2}\right)$ and the differentiability of $u$ w.r.t. $v$ yields

$$
\begin{aligned}
D_{v} u(x, v, t)= & \int_{t}^{T} v(s) d s+\int_{t}^{T} D_{x} \ell(x(s), v(s), s)(s-t)+D_{v} \ell(x(s), v(s), s) d s \\
& +D_{x} g(x(T), v(T))(T-t)+D_{v} g(x(T), v(T)) .
\end{aligned}
$$

By (3.29) and (3.24), we obtain

$$
\begin{aligned}
\int_{t}^{T} D_{x} \ell(x(s), v(s), s)(s-t) d s & =\int_{t}^{T} p_{x}^{\prime}(s)(s-t) d s=p_{x}(T)(T-t)-\int_{t}^{T} p_{x}(s) d s \\
& =-D_{x} g(x(T), v(T))(T-t)-\int_{t}^{T} p_{x}(s) d s .
\end{aligned}
$$

Hence

$$
\begin{aligned}
D_{v} u(x, v, t) & =\int_{t}^{T}\left(v(s)-p_{x}(s)+D_{v} \ell(x(s), v(s), s)\right) d s+D_{v} g(x(T), v(T)) \\
& =\int_{t}^{T} p_{v}^{\prime}(s) d s+D_{v} g(x(T), v(T)) \\
& =-p_{v}(t)=-\alpha(t),
\end{aligned}
$$

where the last two equalities are due to $(3.30),(3.26)$ and the terminal condition for $p$. This uniquely determines the value of $\alpha(\cdot)$ at time $t$.

Conversely we prove that, if all $\alpha(\cdot) \in \mathcal{U}(x, v, t)$ take the same value $\alpha(t)$ at $t$, then $D_{v} u(x, v, t)$ exists. Fix $x$ and $t$. From the semi-concavity of $u(x, \cdot, t)$, the differentiability of $u(x, \cdot, t)$ at $v$ will follow from the fact that $D_{v}^{*} u(x, v, t)$ is a singleton (see [11, Proposition 3.3.4]). Recall that the set of reachable gradients of $u(x, \cdot, t)$ is defined by

$$
D_{v}^{*} u(x, v, t)=\left\{\begin{array}{l|l}
\chi \in \mathbb{R}^{N}: \exists\left(v_{n}\right)_{n \in \mathbb{N}} \text { with } \mid \begin{array}{l}
\lim _{n \rightarrow \infty} v_{n}=v \\
u(x, \cdot, t) \text { is differentiable at } v_{n}, \\
\lim _{n \rightarrow \infty} D_{v} u\left(x, v_{n}, t\right)=\chi .
\end{array}
\end{array} .\right.
$$

Take $\chi \in D_{v}^{*} u(x, v, t)$. By definition of $D_{v}^{*} u(x, v, t)$ there exist sequences $\left\{v_{n}\right\},\left\{\chi_{n}=\right.$ $\left.D_{v} u\left(x, v_{n}, t\right)\right\}$ such that

$$
v_{n} \rightarrow v \text { and } \quad \chi_{n} \rightarrow \chi
$$


Consider $\alpha_{n} \in \mathcal{U}\left(x, v_{n}, t\right)$; by the other part of the statement (already proven), we know that

$$
-\alpha_{n}(t)=D_{v} u\left(x, v_{n}, t\right)=\chi_{n}
$$

From estimate (3.33) in Corollary 3.1, we see that

$$
\left\|\alpha_{n}^{\prime}\right\|_{\infty} \leqslant C\left(1+\left|v_{n}\right|\right) \leqslant C, \text { for any } n .
$$

Hence from Ascoli-Arzelà Theorem, we deduce that, after extracting a subsequence, $\alpha_{n}$ uniformly converge to some $\alpha \in C\left([t, T] ; \mathbb{R}^{N}\right)$. In particular, calling $\left(x_{n}(\cdot), v_{n}(\cdot)\right)$ the trajectory associated to $\alpha_{n}$ starting from $\left(x, v_{n}\right)$ :

$$
x_{n}(s)=x+v_{n}(s-t)+\int_{t}^{s} \int_{t}^{\theta} \alpha_{n}(\tau) d \tau d \theta, \quad \text { and } \quad v_{n}(s)=v_{n}+\int_{t}^{s} \alpha_{n}(\tau) d \tau .
$$

we get:

$$
\begin{aligned}
& x_{n}(s) \rightarrow x(s)=x+v(s-t)+\int_{t}^{s} \int_{t}^{\theta} \alpha(\tau) d \tau d \theta, \text { uniformly in }[t, T], \\
& v_{n}(s) \rightarrow v(s)=v+\int_{t}^{s} \alpha(\tau) d \tau \text { uniformly in }[t, T] .
\end{aligned}
$$

Moreover, by classical arguments of stability, $\alpha$ is optimal, i.e. $\alpha \in \mathcal{U}(x, v, t)$. The uniform convergence of the $\alpha_{n}$ yields in particular that $\alpha_{n}(t) \rightarrow \alpha(t)$ where $\alpha(t)$ is uniquely determined by assumption. By (3.35) and (3.36), we get that $\chi_{n} \rightarrow \chi=\alpha(t)$. This implies that $D_{v}^{*} u(x, v, t)$ is a singleton, then $D_{v} u(x, v, t)$ exists. Going back to the first part of the proof, we see that $D_{v} u(x, v, t)=-\alpha(t)$.

2. If $\mathcal{U}(x, v, t)=\{\alpha(\cdot)\}$, then for any $s \in[t, T], \alpha(s)$ is uniquely determined. Indeed, if there exists $\beta \in \mathcal{U}(x(s), v(s), s)$, then the concatenation $\gamma$ of $\alpha$ and $\beta$ (see Lemma 3.1) is also optimal, i.e. $\gamma \in \mathcal{U}(x, v, t)=\{\alpha(\cdot)\}$.

Then from point 1 with $t=s$ at $(x(s), v(s))$, we deduce that $D_{v} u(x(s), v(s), s)$ exists.

3. From point 1 , we know that for any $\alpha(\cdot) \in \mathcal{U}(x, v, t), \alpha(t)$ is unique and coincides with $-D_{v} u(x, v, t)$. Hence, relation (3.26) ensures $p_{v}(t)=-D_{v} u(x, v, t)$. On the other hand, note that, since $D_{x} u(x, v, t)$ exists, we get from (3.34) that

$$
\begin{aligned}
D_{x} u(x, v, t) & =\int_{t}^{T} D_{x} \ell(x(s), v(s), s) d s+D_{x} g(x(T), v(T)) \\
& =\int_{t}^{T} p_{x}^{\prime}(s) d s+D_{x} g(x(T), v(T))=-p_{x}(t) ;
\end{aligned}
$$

thus, $p_{x}(t)$ and $p_{v}(t)$ are both uniquely determined. Hence (3.27)-(3.30) is a system of differential equations with initial conditions $x(t), v(t), p_{x}(t)$ and $p_{v}(t)$ which admits a unique solution $\left(x(\cdot), v(\cdot), p_{x}(\cdot), p_{v}(\cdot)\right)$ by Cauchy-Lipschitz theorem, and $(x(\cdot), v(\cdot))$ is the unique optimal trajectory starting from $(x, v)$, associated to the unique optimal control law $\alpha(\cdot)=p_{v}(\cdot)$.

Lemma 3.5 (optimal synthesis). Consider $\xi \in \mathbb{R}^{N}$ and $\eta \in \mathbb{R}^{N}$. 
1. Let $x \in C^{1}\left([t, T] ; \mathbb{R}^{N}\right), v \in \mathrm{AC}\left([t, T] ; \mathbb{R}^{N}\right)$ be such that

$$
x(t)=\xi, \quad \text { and } \quad v(t)=\eta,
$$

and for almost every $s \in(t, T)$,

$$
u(x(s), \cdot, s) \text { is differentiable at } v(s),
$$

and

$$
\begin{aligned}
& x^{\prime}(s)=v(s), \\
& v^{\prime}(s)=-D_{v} u(x(s), v(s), s),
\end{aligned}
$$

where $u$ is the solution of (3.4). Under these assumptions, the control law $\alpha(s)=$ $v^{\prime}(s)=-D_{v} u(x(s), v(s), s)$ is optimal for $u(\xi, \eta, t)$.

2. If $u(\cdot, \cdot, t)$ is differentiable at $(\xi, \eta)$, then problem (3.38), (3.40) has a unique solution corresponding to the optimal trajectory.

Proof. We adapt the arguments of [12, Lemma 4.11]. Fix $(t, \xi, \eta) \in(0, T) \times \mathbb{R}^{2 N}$. Let $x \in C^{1}\left([t, T] ; \mathbb{R}^{N}\right), v \in \mathrm{AC}\left([0, T] ; \mathbb{R}^{N}\right)$ be as in the statement. Note that, from (3.40), since $\left|D_{v} u\right|$ grows at most linearly in $v$ (see Lemma 3.2-(point 1)), Gronwall's Lemma ensures that $v(\cdot)$ is bounded in $(t, T)$; consequently, again by (3.40) and Lemma 3.2-(point 1$), v(\cdot)$ is Lipschitz continuous. Therefore, from Lemma 3.2, the function $s \mapsto u(x(s), v(s), s)$ is Lipschitz continuous as well. Hence, for almost every $s \in[t, T]$,

- (3.39) and (3.40) hold,

- the function $u(x(\cdot), v(\cdot), \cdot)$ admits a derivative at $s$.

Fix such an s. Lebourg's Theorem for Lipschitz functions (see [17, Thm 2.3.7] and [17, Thm 2.5.1]) ensures that, for any sufficiently small number $h$, there exists $\left(y_{h}, w_{h}, s_{h}\right)$ in the open line segment $((x(s), v(s), s),(x(s+h), v(s+h), s+h))$ and $\left(\chi_{x}^{h}, \chi_{v}^{h}, \chi_{t}^{h}\right) \in$ $\operatorname{conv}\left(D_{x, v, t}^{*} u\left(y_{h}, w_{h}, s_{h}\right)\right)$ such that

$u(x(s+h), v(s+h), s+h)-u(x(s), x(s), s)=\chi_{x}^{h} \cdot(x(s+h)-x(s))+\chi_{v}^{h} \cdot(v(s+h)-v(s))+\chi_{t}^{h} h$.

Here, $\operatorname{conv}(\mathrm{A})$ stands for the convex hull of a set $A$ while $D_{x, v, t}^{*} u\left(y_{h}, w_{h}, s_{h}\right)$ stands for the reachable gradient at $\left(y_{h}, w_{h}, s_{h}\right)$ with respect to the variables $x, v$ and $t$ (see [4, eq. (4.4)]. By Carathéodory's theorem (see [11, Thm A.1.6]), there exist $\left(\lambda^{h, i}, \chi_{x}^{h, i}, \chi_{v}^{h, i}, \chi_{t}^{h, i}\right)_{i=1, \ldots, 2 N+2}$ such that $\lambda^{h, i} \geqslant 0, \sum_{i=1}^{2 N+2} \lambda^{h, i}=1,\left(\chi_{x}^{h, i}, \chi_{v}^{h, i}, \chi_{t}^{h, i}\right) \in D_{x, v, t}^{*} u\left(y_{h}, w_{h}, s_{h}\right)$ and $\left(\chi_{x}^{h}, \chi_{v}^{h}, \chi_{t}^{h}\right)=$ $\sum_{i=1}^{2 N+2} \lambda^{h, i}\left(\chi_{x}^{h, i}, \chi_{v}^{h, i}, \chi_{t}^{h, i}\right)$. We claim that, for any $i=1, \ldots, 2 N+2$, there holds

$$
\lim _{h \rightarrow 0} \chi_{v}^{h, i}=D_{v} u(x(s), v(s), s) .
$$

Indeed, let $\chi_{v}^{i}$ be any cluster point of $\left\{\chi_{v}^{h, i}\right\}_{h}$. After a diagonal extraction, there exist $\left(x_{n}, v_{n}, t_{n}\right)$ such that $u$ is differentiable at $\left(x_{n}, v_{n}, t_{n}\right),\left(x_{n}, v_{n}, t_{n}\right) \rightarrow(x(s), v(s), s)$ and $D_{v} u\left(x_{n}, v_{n}, t_{n}\right) \rightarrow \chi_{v}^{i}$ as $n \rightarrow \infty$. By [12, Lemma 4.6] (applied to $\left.z_{n}(\cdot):=u\left(x_{n}, \cdot, t_{n}\right)\right)$, we have

$$
\chi_{v}^{i}=\lim _{n} D_{v} u\left(x_{n}, v_{n}, t_{n}\right) \in D^{+} z(v(s))
$$

where $z(\cdot):=u(x(s), \cdot, s)$. On the other hand, assumption (3.39) ensures that $z$ is differentiable at $v(s)$; hence, by [11, Proposition 3.1.5-(c)], we get $\chi_{v}^{i}=D_{v} u(x(s), v(s), s)$ and 
our claim is proved. In particular, we deduce that $\chi_{v}^{h}$ converge to $D_{v} u(x(s), v(s), s)$ as $h \rightarrow 0$.

On the other hand, since $u$ is a viscosity solution to equation (3.4) and $\left(\chi_{x}^{h, i}, \chi_{v}^{h, i}, \chi_{t}^{h, i}\right) \in$ $D_{x, v, t}^{*} u\left(y_{h}, w_{h}, s_{h}\right)$, we obtain that for all $i \in 1, \ldots, 2 N+2$,

$$
-\chi_{t}^{h, i}+\frac{1}{2}\left|\chi_{v}^{h, i}\right|^{2}-\frac{1}{2}\left|w_{h}\right|^{2}-w_{h} \cdot \chi_{x}^{h, i}=\ell\left(y_{h}, w_{h}, s_{h}\right) .
$$

Therefore, $\chi_{t}^{h}+w_{h} \cdot \chi_{x}^{h}=\frac{1}{2} \sum_{i=1}^{2 N+2} \lambda^{h, i}\left|\chi_{v}^{h, i}\right|^{2}-\frac{1}{2}\left|w_{h}\right|^{2}-\ell\left(y_{h}, w_{h}, s_{h}\right)$ converges to $\frac{1}{2}\left|D_{v} u(x(s), v(s), s)\right|^{2}-\frac{1}{2}|v(s)|^{2}-\ell(x(s), v(s), s)$ as $h \rightarrow 0$.

Then dividing (3.41) by $h$ and letting $h$ tend to 0 , we get that

$$
\begin{aligned}
& \frac{d}{d s}(u(x(s), v(s), s)) \\
= & D_{v} u(x(s), v(s), s) \cdot v^{\prime}(s)+\frac{1}{2}\left|D_{v} u(x(s), v(s), s)\right|^{2}-\frac{1}{2}|v(s)|^{2}-\ell(x(s), v(s), s) .
\end{aligned}
$$

Recalling (3.40), we get

$$
\frac{d}{d s}(u(x(s), v(s), s))=-\frac{1}{2}\left|D_{v} u(x(s), v(s), s)\right|^{2}-\frac{1}{2}|v(s)|^{2}-\ell(x(s), v(s), s) .
$$

or in equivalent manner,

$$
\frac{d}{d s}(u(x(s), v(s), s))=-\frac{1}{2}\left|v^{\prime}(s)\right|^{2}-\frac{1}{2}|v(s)|^{2}-\ell(x(s), v(s), s),
$$

which holds for almost every $s$. Integrating this equality on $[t, T]$ and taking into account the terminal condition in (3.4), we obtain

$$
u(x, v, t)=\int_{t}^{T} \frac{1}{2}\left|v^{\prime}(s)\right|^{2}+\frac{1}{2}|v(s)|^{2}+\ell(x(s), v(s), s) d s+g(x(T), v(T)) .
$$

Therefore, the control law $\alpha(s)=v^{\prime}(s)=-D_{v} u(x(s), v(s), s)$ is optimal. This achieves the proof of the first statement.

The second statement is a direct consequence of Lemma 3.4.

\section{The continuity equation}

In this section, our aim is to study equation (2.1)-(ii), and more precisely the wellposedness of

$$
\begin{cases}\partial_{t} m+v \cdot D_{x} m-\operatorname{div}_{v}\left(m D_{v} u\right)=0, & \text { in } \mathbb{R}^{2 N} \times(0, T), \\ m(x, v, 0)=m_{0}(x, v), & \text { on } \mathbb{R}^{2 N}\end{cases}
$$

where $u$ is the value function associated to the cost $J_{t}$ in (3.3); for the sake of clarity, let us recall from Proposition 3.1 that $u$ is the unique viscosity solution fulfilling (3.11) to the problem

$$
\begin{cases}-\partial_{t} u-v \cdot D_{x} u+\frac{1}{2}\left|D_{v} u\right|^{2}-\frac{1}{2}|v|^{2}-l(x, v)=F[\bar{m}(t)](x, v), & \text { in } \mathbb{R}^{2 N} \times(0, T), \\ u(x, v, T)=G[\bar{m}(T)](x, v), & \text { on } \mathbb{R}^{2 N},\end{cases}
$$


and $\bar{m}$ is fixed and belongs to $C\left([0, T] ; \mathcal{P}_{1}\left(\mathbb{R}^{2 N}\right)\right)$. It is worth to observe that the differential equation in (4.1) can also be written

$$
\partial_{t} m-\operatorname{div}_{x, v}(m b)=0,
$$

with $b:=\left(-v, D_{v} u\right)$. In the present framework, the properties of $u$ (semi-concavity and local Lipschitz continuity) are not enough to ensure that the flow $\Phi(x, t, s)$ given by Lemma 3.5 has a Lipschitz continuous inverse, by contrast with [12, Lemma 4.13]. Moreover, the drift $b$ is only locally bounded; this lack of regularity makes it impossible to apply the standard results for drifts which are Lipschitz continuous (uniqueness, existence and representation formula of $m$ as the push-forward of $m_{0}$ through the characteristic flow; e.g., see [2, Proposition 8.1.8]). We shall overcome this difficulty by applying the superposition principle [2, Theorem 8.2.1]. The latter yields a representation formula of $m$ as the push-forward of some measure on $C\left([0, T] ; \mathbb{R}^{2 N}\right)$ through the evaluation map $e_{t}$ defined by $e_{t}(\gamma)=\gamma(t)$ for all continuous function $\gamma$ with value in $\mathbb{R}^{2 N}$. In the following theorem, we state existence, uniqueness, and some regularity results for (4.1):

Theorem 4.1. Under assumptions $(\mathrm{H})$, for any $\bar{m} \in C\left([0, T] ; \mathcal{P}_{1}\left(\mathbb{R}^{2 N}\right)\right)$, there is a unique $m \in C^{\frac{1}{2}}\left([0, T] ; \mathcal{P}_{1}\left(\mathbb{R}^{2 N}\right)\right) \cap L^{\infty}\left((0, T) ; \mathcal{P}_{2}\left(\mathbb{R}^{2 N}\right)\right)$ which solves problem $(4.1)$ in the sense of Definition 2.1.

Moreover $m(t, \cdot)$ satisfies: for any for $\phi \in C_{b}^{0}\left(\mathbb{R}^{2 N}\right)$, for any $t \in[0, T]$,

$$
\int_{\mathbb{R}^{2 N}} \phi(x, v) m(x, v, t) d x d v=\int_{\mathbb{R}^{2 N}} \phi\left(\bar{\gamma}_{x, v}(t)\right) m_{0}(x, v) d x d v,
$$

where, for a.e. $(x, v) \in \mathbb{R}^{2 N}, \bar{\gamma}_{x, v}$ is the solution to $(2.2)$.

The proof of Theorem 4.1 is given in the next two subsections which are devoted respectively to existence (see Proposition 4.1) and to uniqueness and the representation formula (see Proposition 4.2).

\subsection{Existence of the solution}

We wish to establish the existence of a solution to the continuity equation via a vanishing viscosity method applied to the whole MFG system in which the viscous terms involve Laplace operators with respect to both $x$ and $v$. This is reminiscent of [13, Appendix] (see also [12, Section 4.4]). In this way, $D_{v} u$ is replaced by $D_{v} u^{\sigma}$, which is regular by standard regularity theory for parabolic equations; this implies the regularity of the solution of the Fokker-Planck equation (see [15]). Note also that $D_{v} u$ may be unbounded; we shall overcome this issue by taking advantage of estimates similar to those in Lemma 3.2. Indeed, these estimates will allow us to apply classical results for the existence and uniqueness of the solution.

Proposition 4.1. Under assumptions $(\mathrm{H})$, for any $\bar{m} \in C\left([0, T] ; \mathcal{P}_{1}\left(\mathbb{R}^{2 N}\right)\right)$, problem $(4.1)$ has a solution $m$ in the sense of Definition 2.1. Moreover $m \in C^{\frac{1}{2}}\left([0, T] ; \mathcal{P}_{1}\left(\mathbb{R}^{2 N}\right)\right) \cap$ $L^{\infty}\left(0, T ; \mathcal{P}_{2}\left(\mathbb{R}^{2 N}\right)\right)$.

$$
\begin{cases}(i)-\partial_{t} u-\sigma \Delta_{x, v} u-v \cdot D_{x} u+\frac{1}{2}\left|D_{v} u\right|^{2}-\frac{1}{2}|v|^{2}-l(x, v)=F[\bar{m}](x, v), & \text { in } \mathbb{R}^{2 N} \times(0, T), \\ (\text { ii }) \partial_{t} m-\sigma \Delta_{x, v} m-\operatorname{div}_{v}\left(m D_{v} u\right)-v \cdot D_{x} m=0, & \text { in } \mathbb{R}^{2 N} \times(0, T), \\ (\text { iii }) m(x, v, 0)=m_{0}(x, v), \quad u(x, v, T)=G[\bar{m}(T)](x, v), & \text { on } \mathbb{R}^{2 N} .\end{cases}
$$


Recall that equation (4.3)-(ii) has a standard probabilistic interpretation (see relation (4.8) below). Our aim is to find a solution to problem (4.1) by letting $\sigma$ tend to $0^{+}$. To this end, some estimates are needed.

Note that equation (4.3)-(ii) can be written in the compact form

$$
\partial_{t} m-\sigma \Delta_{x, v} m-\operatorname{div}_{x, v}\left(m b^{\sigma}\right)=0, \quad \text { with } b^{\sigma}:=\left(-v, D_{v} u\right) .
$$

We start by establishing the well-posedness of system (4.3) and that the functions $u^{\sigma}$ are Lipschitz continuous and semi-concave uniformly in $\sigma$.

Lemma 4.1. Under the same assumptions as in Proposition 4.1, there exists a unique classical solution $u^{\sigma}$ to problem (4.3)-(i), -(iii) with quadratic growth in $(x, v)$. Moreover, there exists a constant $C>0$ which depends only on the constants in assumptions $(\mathrm{H})$, in particular it is independent of $\sigma \leqslant 1$, such that

(a) $\left|u^{\sigma}(x, v, t)\right| \leqslant C\left(1+|v|^{2}\right)$,

(b) $\left\|D_{x} u^{\sigma}\right\|_{\infty} \leqslant C, \quad\left|D_{v} u^{\sigma}(x, v, t)\right| \leqslant C(1+|v|), \quad\left|\partial_{t} u^{\sigma}(x, v, t)\right| \leqslant C\left(1+|v|^{2}\right)$,

(c) $D_{x, v}^{2} u^{\sigma} \leqslant C$,

where $D_{x, v}^{2} u$ is the Hessian of $u$ with respect to both $x$ and $v$.

Proof. Following the same arguments of Proposition 3.1 (based on the comparison principle by Da Lio and Ley [19, Theorem 2.1]), one can easily prove the existence of a viscosity solution to equation (4.3)-(i) with terminal condition as in (4.3)-(iii) and satisfying inequality $(a)$. Furthermore, still by the results in [19], this solution is unique among the functions with this growth at infinity. Hence, estimate $(a)$ is proved.

Let us now prove that this viscosity solution $u^{\sigma}$ is a classical solution. To this end, let us assume for a moment that $u^{\sigma}$ satisfies estimates $(b)$ and $(c)$. We see that $u$ is a viscosity subsolution of

$$
-\partial_{t} u-\sigma \Delta_{x, v} u-v \cdot D_{x} u \leqslant C\left(1+|v|^{2}\right) .
$$

Moreover, from estimate $(c)$, we see that at any point $(x, v, t)$, either $u^{\sigma}$ is twice differentiable with respect to $x$ and $v$, or there does exist a smooth function that touches $u^{\sigma}$ from below. This and estimate (b) imply that $u^{\sigma}$ is a viscosity supersolution of

$$
-\partial_{t} u-\sigma \Delta_{x, v} u-v \cdot D_{x} u \geqslant-C\left(1+|v|^{2}\right) .
$$

for some positive constant $C$. From [22], $u$ is also a distributional subsolution (respectively supersolution) of the same linear inequalities. Therefore, both $-\partial_{t} u^{\sigma}-\sigma \Delta_{x, v} u^{\sigma}-v \cdot D_{x} u^{\sigma}$ and $-\frac{1}{2}\left|D_{v} u^{\sigma}\right|^{2}+\frac{1}{2}|v|^{2}+l(x, v)+F[\bar{m}](x, v)$ are in $L_{\mathrm{loc}}^{\infty}$. On the other hand, from $(b)$ and (c), Alexandrov's theorem implies that $u^{\sigma}$ is twice differentiable with respect to $x$ and $v$ almost everywhere, so the equation

$$
-\partial_{t} u^{\sigma}-\sigma \Delta_{x, v} u^{\sigma}-v \cdot D_{x} u^{\sigma}=-\frac{1}{2}\left|D_{v} u^{\sigma}\right|^{2}+\frac{1}{2}|v|^{2}+\ell(x, v, t),
$$

(where $\ell$ and $g$ are defined in (3.1)), holds almost everywhere, and in the sense of distributions since both the left and right hand sides are in $L_{\text {loc }}^{\infty}$.

Hence classical results on the regularity of weak solutions (including bootstrap) can be applied and yield that $u$ is a classical solution. 
Let us now prove the estimates $(b)$ and $(c)$, by using similar arguments to those contained in the proofs of Lemma 3.2. They use a representation formula of $u$ arising from a stochastic optimal control problem (see, for example, [19, 6, 12]).

Let $\left(\Omega, \mathcal{F},\left(\mathcal{F}_{t}\right), \mathbb{P}\right)$ be a complete filtered probability space, the filtration $\left(\mathcal{F}_{t}\right)$ supporting a standard $2 N$-dimensional Brownian motion $B_{s}=\left(B_{x, s}, B_{v, s}\right)$. Let $\mathcal{A}_{t}$ be the set of $\mathbb{R}^{N_{-}}$ valued $\left(\mathcal{F}_{t}\right)$-progressively measurable processes and let $\mathbb{E}$ be the expectation with respect to the probability measure $\mathbb{P}$. The unique solution of (4.3)-(i) which satisfies point (a) can be written as:

$u^{\sigma}(x, v, t)=\inf _{\alpha \in \mathcal{A}_{t}} \mathbb{E}\left(\int_{t}^{T}\left[\frac{1}{2}|\alpha(s)|^{2}+\frac{1}{2}|V(s)|^{2}+\ell(X(s), V(s), s)\right] d s+g(X(T), V(T))\right)$

where the controlled process $(X(\cdot), V(\cdot))$ satisfies

$$
X(t)=x, \quad V(t)=v,
$$

almost surely and is governed by the stochastic differential equations

$$
\left\{\begin{array}{l}
d X=V(s) d s+\sqrt{2 \sigma} d B_{x, s} \\
d V=\alpha(s) d s+\sqrt{2 \sigma} d B_{v, s}
\end{array}\right.
$$

Thus, almost surely,

$$
\left\{\begin{array}{l}
X(s)=x+v(s-t)+\int_{t}^{s} \int_{t}^{\theta} \alpha(\tau) d \tau d \theta+\sqrt{2 \sigma} \int_{t}^{s}\left(\int_{t}^{\theta} d B_{v, \tau}\right) d \theta+\sqrt{2 \sigma} \int_{t}^{s} d B_{x, \tau}, \\
V(s)=v+\int_{t}^{s} \alpha(\tau) d \tau+\sqrt{2 \sigma} \int_{t}^{s} d B_{v, \tau} .
\end{array}\right.
$$

To prove $(b)$, we can exactly use the same arguments as for Lemma 3.2, replacing the paths $(x(s), v(s))$ and $(y(s), w(s))$ by the processes $(X(s), V(s))$ and $(Y(s), W(s))$, and noting that, from (4.6), we get similar equalities as in (3.8).

Note that, for any $\sigma$, we get from $(a)$ that any $\epsilon$-optimal control $\alpha^{\sigma}$ for $u^{\sigma}(x, v, t)$ satisfies

$$
\mathbb{E}\left(\int_{t}^{T}\left|\alpha^{\sigma}(s)\right|^{2} d s\right) \leqslant C\left(1+|v|^{2}\right)
$$

hence, we get the same estimates as (3.9) and (3.10), namely estimate (b). An analytic proof of $(b)$ is also possible, see [27, Chapter XI].

In order to prove $(c)$, we can follow the same procedure as in the proof of Lemma 3.3, noting that:

i) equalities (3.14) and (3.18) are still true for the stochastic processes, ii) if we fix $s \in[t, T]$, using a Taylor expansion of $g$ as in (3.16), we get

$$
\begin{aligned}
& g(X(s), V(s))=g\left(X_{\lambda}(s), V_{\lambda}(s)\right)+D g\left(X_{\lambda}(s), V_{\lambda}(s)\right)\left(X(s)-X_{\lambda}(s), V(s)-V_{\lambda}(s)\right) \\
& +\frac{1}{2}\left(X(s)-X_{\lambda}(s), V(s)-V_{\lambda}(s)\right) D^{2} g(\xi, \eta)\left(X(s)-X_{\lambda}(s), V(s)-V_{\lambda}(s)\right)^{T},
\end{aligned}
$$

where $\xi=X(s)+\theta_{1}\left(X(s)-X_{\lambda}(s)\right), \eta=V(s)+\theta_{2}\left(V(s)-V_{\lambda}(s)\right)$ for suitable $\theta_{1}$ and $\theta_{2}$ in $[0,1]$. For a similar proof, see $[6]$. 
Lemma 4.2. Under the same assumptions as in Proposition 4.1, there exists a unique classical solution $m^{\sigma}$ to problem (4.3)-(ii), -(iii) with a sub-exponential growth in $(x, v)$. Moreover, $m^{\sigma}>0$.

Proof. By Lemma 4.1, the problem for $m^{\sigma}$ can be written

$$
\partial_{t} m-\sigma \Delta_{x, v} m-b^{\sigma} \cdot D_{x v} m-\left(\Delta_{v} u^{\sigma}\right) m=0, \quad m(0)=m_{0},
$$

where $b^{\sigma}$ has been introduced in (4.4) and from the estimates contained in Lemma 4.1, $\left|b^{\sigma}\right| \leqslant C(1+|v|)$ and $\Delta_{v} u^{\sigma} \leqslant C$. Using this and the results contained in [21], we get the existence and uniqueness of a classical solution $m^{\sigma}$ of (4.3)-(ii) with initial condition as in (4.3)-(iii). From the assumptions on $m_{0}$ and Harnack inequality (see for example [23, Theorem 2.1, p.13]) we get that $m^{\sigma}(\cdot, t)>0$ for $t>0$.

Let us now prove some properties of the functions $m^{\sigma}$ which will play a crucial role in the proofs of Proposition 4.1 and of Theorem 2.1.

Lemma 4.3. Under the same assumptions of Proposition 4.1, there exists a constant $K>0$ which depends only on the constants in assumptions $(\mathrm{H})$ and on $m_{0}$, in particular it is independent of $\sigma \leqslant 1$, such that:

1. $\left\|m^{\sigma}\right\|_{\infty} \leqslant K$

2. $\quad \mathbf{d}_{1}\left(m^{\sigma}\left(t_{1}\right), m^{\sigma}\left(t_{2}\right)\right) \leqslant K\left(t_{2}-t_{1}\right)^{1 / 2}, \quad \forall t_{1} \leqslant t_{2} \in[0, T]$

3. $\quad \int_{\mathbb{R}^{2 N}}\left(|x|^{2}+|v|^{2}\right) d m^{\sigma}(t)(x, v) \leqslant K\left(\int_{\mathbb{R}^{2 N}}\left(|x|^{2}+|v|^{2}\right) d m_{0}(x, v)+1\right), \quad \forall t \in[0, T]$.

Proof. Point 1. In order to prove this $L^{\infty}$ estimate, we argue as in [13, Theorem 5.1]. We note that

$$
\operatorname{div}_{v}\left(m^{\sigma} D_{v} u^{\sigma}\right)=D_{v} m^{\sigma} \cdot D_{v} u^{\sigma}+m^{\sigma}\left(\Delta_{v} u^{\sigma}\right) \leqslant D_{v} m^{\sigma} \cdot D_{v} u^{\sigma}+C m^{\sigma},
$$

because of the semi-concavity of $u$ established in Lemma 4.1 and the positivity of $m^{\sigma}$. Therefore, from assumption (H2), the function $m^{\sigma}$ satisfies

$$
\partial_{t} m^{\sigma}-\sigma \Delta_{x, v} m^{\sigma}-v \cdot D_{x} m^{\sigma}-D_{v} u^{\sigma} \cdot D_{v} m^{\sigma}-C m^{\sigma} \leqslant 0, \quad m^{\sigma}(x, v, 0) \leqslant C .
$$

Then, using $w=C e^{C t}$ as a supersolution (recall that $C$ is independent of $\sigma$ ), we obtain that $\left\|m^{\sigma}\right\|_{\infty} \leqslant C e^{C T}$, using the comparison principle proved in [19, Theorem 2.1].

To prove Points 2 and 3 as in the proof of [12, Lemma 3.4 and 3.5], it is convenient to introduce the stochastic differential equation

$$
d Y_{t}=b^{\sigma}\left(Y_{t}, t\right) d t+\sqrt{2 \sigma} d B_{t}, \quad Y_{0}=Z_{0},
$$

where $Y_{t}=\left(X_{t}, V_{t}\right), b^{\sigma}(x, v, t)=\left(-v, D_{v} u^{\sigma}(x, v, t)\right), B_{t}$ is a standard $2 N$-dimensional Brownian motion, and $\mathcal{L}\left(Z_{0}\right)=m_{0}$. By standard arguments, setting

$$
m^{\sigma}(t):=\mathcal{L}\left(Y_{t}\right)
$$

we know that $m^{\sigma}(t)$ is absolutely continuous with respect to Lebesgue measure, and that if $m^{\sigma}(\cdot, \cdot, t)$ is the density of $m^{\sigma}(t)$, then $m^{\sigma}$ is the weak solution to (4.3)-(ii) with $\left.m^{\sigma}\right|_{t=0}=m_{0}$ (from Ito's Theorem, since $b^{\sigma}$ has at most linear growth with respect to $(x, v)$, Proposition 3.6 Chapter 5 [20], p.303, and the book [5]). Here again, we have used the estimate on $\left|D_{v} u^{\sigma}\right|$ given in Lemma 4.1. 
Point 3: Noting that

$$
\int_{\mathbb{R}^{2 N}}\left(|x|^{2}+|v|^{2}\right) d m^{\sigma}(t)(x, v)=\mathbb{E}\left(\left|Y_{t}\right|^{2}\right),
$$

the desired estimate can be obtained by applying Estimate 3.17 of Problem 3.15, p. 306, (the solutions are at p. 389) of [20] with $m=1$.

Point 2: For $t_{2} \geqslant t_{1}$, it is well known that

$$
\mathbf{d}_{1}\left(m^{\sigma}\left(t_{1}\right), m^{\sigma}\left(t_{2}\right)\right) \leqslant \mathbb{E}\left(\left|Y_{t_{1}}-Y_{t_{2}}\right|\right) .
$$

Recall also that for a suitable constant $C$,

$$
\left|b^{\sigma}\left(Y_{\tau}, \tau\right)\right| \leqslant C\left(\left|V_{\tau}\right|+1\right) .
$$

The latter two observations imply that

$$
\begin{aligned}
\mathbb{E}\left(\left|Y_{t_{1}}-Y_{t_{2}}\right|\right) & \leqslant \mathbb{E}\left(\int_{t_{1}}^{t_{2}}\left|b^{\sigma}\left(Y_{\tau}, \tau\right)\right| d \tau+\sqrt{2 \sigma}\left|B_{t_{2}}-B_{t_{1}}\right|\right) \\
& \leqslant \mathbb{E}\left(C \int_{t_{1}}^{t_{2}}\left(\left|V_{\tau}\right|+1\right)|d \tau+\sqrt{2 \sigma}| B_{t_{2}}-B_{t_{1}} \mid\right) \\
& \leqslant C\left(\mathbb{E}\left(\int_{t_{1}}^{t_{2}}\left(\left|V_{\tau}\right|^{2}+1\right) \mid d \tau\right)\right)^{\frac{1}{2}} \sqrt{t_{2}-t_{1}}+\sqrt{2 \sigma} \sqrt{t_{2}-t_{1}} \\
& \leqslant C\left(\mathbb{E}\left(\max _{\left[t_{1}, t_{2}\right]}\left|Y_{\tau}\right|^{2}\right)+1\right)^{\frac{1}{2}}\left(t_{2}-t_{1}\right)+\sqrt{2 \sigma} \sqrt{t_{2}-t_{1}} .
\end{aligned}
$$

where we have used estimate [20, (3.17) p. 306].

Proof of Proposition 4.1. The arguments are similar to those in the proof of $[13$, Theorem 5.1] (see also [12, Theorem 4.20]). Lemma 4.1 implies that possibly after the extraction of a subsequence, $u^{\sigma}$ locally uniformly converges to some function $u$, which is Lipschitz continuous with respect to $x$, locally Lipschitz continuous with respect to $v$, and $D u^{\sigma} \rightarrow$ $D u$ a.e. (because of the semi-concavity estimate of Lemma 4.1 and [11, Theorem 3.3.3]). By standard stability result for viscosity solutions, the function $u$ is a viscosity solution of (3.4).

On the other hand, the function $m^{\sigma}$ satisfies the estimates stated in Lemma 4.3:

1. from point $3, m^{\sigma}(t)$ is bounded in $\mathcal{P}_{2}\left(\mathbb{R}^{2 N}\right)$ uniformly in $\sigma \in[0,1]$ and $t \in[0, T]$

2. from points 2 and $3, m^{\sigma}$ is bounded in $C^{1 / 2}\left([0, T] ; \mathcal{P}_{1}\left(\mathbb{R}^{2 N}\right)\right)$ uniformly with respect to $\sigma \in[0,1]$.

Recalling that the subsets of $\mathcal{P}_{1}\left(\mathbb{R}^{2 N}\right)$ whose elements have uniformly bounded second moment are relatively compact in $\mathcal{P}_{1}\left(\mathbb{R}^{2 N}\right)$, see for example [12, Lemma 5.7], we can apply Ascoli-Arzelà theorem: we may extract a sequence (still indexed by $\sigma$ for simplicity) such that $\sigma \rightarrow 0^{+}$and $m^{\sigma}$ converges to some $m \in C^{1 / 2}\left([0, T] ; \mathcal{P}_{1}\left(\mathbb{R}^{2 N}\right)\right)$ in the $C\left([0, T] ; \mathcal{P}_{1}\left(\mathbb{R}^{2 N}\right)\right)$ topology. Moreover, from point 1 in Lemma 4.3 and BanachAlaoglu theorem, $m$ belongs to $L_{\text {loc }}^{\infty}\left((0, T) \times \mathbb{R}^{2 N}\right)$ and the sequence $m^{\sigma}$ converges to $m$ in $L_{\text {loc }}^{\infty}\left((0, T) \times \mathbb{R}^{2 N}\right)$-weak-*. 
Therefore, by passing to the limit, we immediately obtain that $\left.m\right|_{t=0}=m_{0},\|m\|_{\infty} \leqslant K$ and that $\mathbf{d}_{1}\left(m\left(t_{1}\right), m\left(t_{2}\right)\right) \leqslant K\left(t_{2}-t_{1}\right)^{1 / 2}, \forall t_{1} \leqslant t_{2} \in[0, T]$.

Let us prove that for all $t \in[0, T]$,

$$
\int_{\mathbb{R}^{2 N}}\left(|x|^{2}+|v|^{2}\right) d m(t)(x, v) \leqslant K\left(\int_{\mathbb{R}^{2 N}}\left(|x|^{2}+|v|^{2}\right) d m_{0}(x, v)+1\right) .
$$

For that, let us consider the increasing sequence of functions defined on $\mathbb{R}_{+}: \phi_{n}(\rho)=$ $1 \wedge((n+1-\rho) \vee 0)$. We know from point 3 in Lemma 4.3, that for all $t \in[0, T]$,

$$
\int_{\mathbb{R}^{2 N}}\left(|x|^{2}+|v|^{2}\right) \phi_{n}\left(|x|^{2}+|v|^{2}\right) m^{\sigma}(x, v, t) d x d v \leqslant K\left(\int_{\mathbb{R}^{2 N}}\left(|x|^{2}+|v|^{2}\right) d m_{0}(x, v)+1\right) .
$$

For a fixed $n$, we can pass to the limit in (4.10) thanks to the $L_{\text {loc }}^{\infty}\left((0, T) \times \mathbb{R}^{2 N}\right)$-weak-* convergence established above. We obtain:

$$
\int_{\mathbb{R}^{2 N}}\left(|x|^{2}+|v|^{2}\right) \phi_{n}\left(|x|^{2}+|v|^{2}\right) m(x, v, t) d x d v \leqslant K\left(\int_{\mathbb{R}^{2 N}} \mid\left(|x|^{2}+|v|^{2}\right) d m_{0}(x, v)+1\right) .
$$

We then pass to the limit as $n \rightarrow+\infty$ thanks to Beppo Levi monotone convergence theorem, and obtain (4.9).

Finally, $m^{\sigma}$ is a solution to (4.3)-(ii),

$$
\int_{0}^{T} \int_{\mathbb{R}^{2 N}} m^{\sigma}\left(-\partial_{t} \psi-\sigma \Delta_{x, v} \psi+D_{v} \psi \cdot D_{v} u^{\sigma}-v \cdot D_{x} \psi\right) d x d v d t=0
$$

for any $\psi \in C_{c}^{\infty}\left((0, T) \times \mathbb{R}^{2 N}\right)$. Applying the dominated convergence theorem, we infer $D_{v} \psi \cdot D_{v} u^{\sigma} \rightarrow D_{v} \psi \cdot D_{v} u$ in $L^{1}$ as $\sigma \rightarrow 0^{+}$because $D_{v} u^{\sigma}$ are locally bounded (see Lemma 4.1), $D_{v} u^{\sigma} \rightarrow D_{v} u$ a.e. and $\psi$ has a compact support. Letting $\sigma \rightarrow 0^{+}$, we conclude from the $L_{\text {loc }}^{\infty}$-weak-* convergence of $m^{\sigma}$ and the convergence $D u^{\sigma} \rightarrow D u$ a.e. that the function $m$ solves (4.1) in the sense of Definition 2.1.

Remark 4.1. Note that we have just proven that all the estimates on $u^{\sigma}$ contained in Lemma 4.1 hold for $u$. These estimates have also been obtained directly in the proof of Lemma 3.2. Similarly, all the estimates on $m^{\sigma}$ contained in Lemma 4.3 hold for $m$.

\subsection{Uniqueness of the solution}

We now deal with uniqueness for (4.1).

Proposition 4.2. Under assumptions $(\mathrm{H})$, the function $m$ found in Proposition 4.1 is the unique solution to problem (4.1) in the sense of Definition 2.1 such that

$m \in C^{\frac{1}{2}}\left([0, T] ; \mathcal{P}_{1}\left(\mathbb{R}^{2 N}\right)\right) \cap L^{\infty}\left((0, T) ; \mathcal{P}_{2}\left(\mathbb{R}^{2 N}\right)\right)$.

Moreover, $m$ satisfies:

$\int_{\mathbb{R}^{2 N}} \phi(x, v) m(x, v, t) d x d v=\int_{\mathbb{R}^{2 N}} \phi\left(\bar{\gamma}_{x, v}(t)\right) m_{0}(x, v) d x d v, \quad \forall \phi \in C_{b}^{0}\left(\mathbb{R}^{2 N}\right), \forall t \in[0, T]$,

where, for a.e. $(x, v) \in \mathbb{R}^{2 N}, \bar{\gamma}_{x, v}$ is the solution to $(2.2)$. 
Proof of Proposition 4.2. The proof is similar to that of [15, Proposition A.1], which relies on the superposition principle [2, Theorem 8.2.1]. Let $\Gamma_{T}$ denote the set of continuous curves in $\mathbb{R}^{2 N}$, namely $\Gamma_{T}=C\left([0, T] ; \mathbb{R}^{2 N}\right)$. For any $t \in[0, T]$, we introduce the evaluation map: $e_{t}: \mathbb{R}^{2 N} \times \Gamma_{T} \rightarrow \mathbb{R}^{2 N}, e_{t}(x, v, \gamma):=\gamma(t)$. Hereafter, when we write "for a.e." without specifying the measure, we intend "with respect to the Lebesgue measure".

Let $m \in C^{1 / 2}\left([0, T] ; \mathcal{P}_{1}\left(\mathbb{R}^{2 N}\right)\right) \cap L^{\infty}\left((0, T) ; \mathcal{P}_{2}\left(\mathbb{R}^{2 N}\right)\right)$ be a solution of problem (4.1) in the sense of Definition 2.1. Recall the notation $b(x, v, t)=\left(-v, D_{v} u(x, v, t)\right)$. The estimate (8.1.20) in chapter 8 of [2] is fulfilled: indeed,

$$
\begin{aligned}
\int_{0}^{T} \int_{\mathbb{R}^{2 N}}|b(x, v, t)|^{2} d m(t)(x, v) \leqslant & C \int_{0}^{T} \int_{\mathbb{R}^{2 N}}|v|^{2} d m(t)(x, v) \\
& +C \int_{0}^{T} \int_{\mathbb{R}^{2 N}}\left|D_{v} u(x, v, t)\right|^{2} d m(t)(x, v) \leqslant C
\end{aligned}
$$

where the last inequality comes from the estimates on $D_{v} u$ and $m$ in Remark 4.2 (recall that $m(t)$ is a probability measure). Therefore, the assumptions of the superposition principle are fulfilled (see [2, Theorem 8.2.1] and also [2, pag. 182]). The latter and the disintegration theorem (see [2, Theorem 5.3.1]) entail that there exist a probability measure $\eta$ on $\mathbb{R}^{2 N} \times \Gamma_{T}$ and for $m_{0}$-almost every $(x, v) \in \mathbb{R}^{2 N}$, a probability measure on $\eta_{x, v}$ on $\Gamma_{T}$, such that

i) $e_{t} \# \eta=m_{t}$, i.e., for every bounded and continuous real valued function $\psi$ defined on $\mathbb{R}^{2 N}$, for every $t \in[0, T]$,

$$
\int_{\mathbb{R}^{2 N}} \psi(x, v) d m_{t}(x, v)=\int_{\mathbb{R}^{2 N} \times \Gamma_{T}} \psi(\zeta(t)) d \eta(x, v, \zeta) .
$$

In particular, $e_{0} \# \eta=m_{0}$.

ii)

$$
\eta=\int_{\mathbb{R}^{2 N}} \eta_{x, v} d m_{0}(x, v)
$$

i.e. for every bounded Borel function $f: \mathbb{R}^{2 N} \times \Gamma_{T} \rightarrow \mathbb{R}$,

$$
\int_{\mathbb{R}^{2 N} \times \Gamma_{T}} f(x, v, \zeta) d \eta(x, v, \zeta)=\int_{\mathbb{R}^{2 N}}\left(\int_{\Gamma_{T}} f(x, v, \zeta) d \eta_{x, v}(\zeta)\right) d m_{0}(x, v) .
$$

iii) For $m_{0}$-almost every $(x, v) \in \mathbb{R}^{2 N}$, the support of $\eta_{x, v}$ is contained in the set

$$
\left\{\begin{array}{l|l}
\zeta \in \mathrm{AC}\left([0, T] ; \mathbb{R}^{2 N}\right): \zeta(t)=(\xi(t), \eta(t)): & \begin{array}{l}
\xi(0)=x, \eta(0)=v, \\
\xi^{\prime}(t)=\eta(t) \\
\eta^{\prime}(t)=-D_{v} u(\xi(t), \eta(t), t) .
\end{array}
\end{array}\right\} .
$$

Recall that in the present case, $m_{0}$ is absolutely continuous (from assumption (H4)); hence, since for all $t \in[0, T], u(\cdot, \cdot, t)$ is Lipschitz continuous, the optimal synthesis in Lemma 3.5 ensures that for a.e. $(x, v) \in \mathbb{R}^{2 N}$, (3.38)-(3.40) (with $t=0$ in the present context) has a unique solution $\bar{\gamma}_{x, v}$, because it is the optimal trajectory for the cost $J_{t}$. Therefore, for 
a.e. $(x, v) \in \mathbb{R}^{2 N}$, the set in (4.13) is a singleton, or in equivalent manner, $\eta_{x, v}$ coincides with $\delta_{\bar{\gamma}_{x, v}}$. In conclusion, for any function $\psi \in C_{b}^{0}\left(\mathbb{R}^{2 N}\right)$,

$$
\begin{aligned}
\int_{\mathbb{R}^{2 N}} \psi(x, v) m(x, v, t) d x d v & =\int_{\mathbb{R}^{2 N} \times \Gamma_{T}} \psi\left(e_{t}(\zeta)\right) d \eta(x, v, \zeta) \\
& =\int_{\mathbb{R}^{2 N}}\left(\int_{\Gamma_{T}} \psi\left(e_{t}(\zeta)\right) d \eta_{x, v}(\zeta)\right) d m_{0}(x, v) \\
& =\int_{\mathbb{R}^{2 N}} \psi\left(e_{t}\left(\bar{\gamma}_{x, v}\right)\right) d m_{0}(x, v) \\
& =\int_{\mathbb{R}^{2 N}} \psi\left(\bar{\gamma}_{x, v}(t)\right) m_{0}(x, v) d x d v
\end{aligned}
$$

This shows that $m$ is uniquely defined as the image of $m_{0}$ by the flow of (2.2).

Proof of Theorem 4.1. Existence of $m$ comes from Proposition 4.1, uniqueness and the representation formula come from Proposition 4.2.

\section{$5 \quad$ Proof of the main results}

Proof of Theorem 2.1. For point 1, we argue as in the proof of [12, Theorem 4.1]. Consider the set $\mathcal{C}:=\left\{m \in C\left([0, T] ; \mathcal{P}_{1}\left(\mathbb{R}^{2 N}\right)\right) \mid m(0)=m_{0}\right\}$ endowed with the norm of $C\left([0, T] ; \mathcal{P}_{1}\left(\mathbb{R}^{2 N}\right)\right)$ and observe that it is a closed and convex subset of $C\left([0, T] ; \mathcal{P}_{1}\left(\mathbb{R}^{2 N}\right)\right)$. We also introduce a map $\mathcal{T}$ as follows: to any $m \in \mathcal{C}$, we associate the solution $u$ to problem (3.4) with $\bar{m}=m$ and to this $u$ we associate the solution $\mu=: \mathcal{T}(m)$ to problem (4.1) which, by Proposition 4.1 belongs to $\mathcal{C}$. Hence, $\mathcal{T}$ maps $\mathcal{C}$ into itself. We claim that the map $\mathcal{T}$ has the following properties:

(a) $\mathcal{T}$ is a continuous map with respect to the norm of $C\left([0, T] ; \mathcal{P}_{1}\left(\mathbb{R}^{2 N}\right)\right)$

(b) $\mathcal{T}$ is a compact map.

Assume for the moment that these properties are true. In this case, Schauder fixed point Theorem ensures the existence of a fixed point for $\mathcal{T}$, namely a solution to system (2.1). Therefore it remains to prove properties $(a)$ and $(b)$.

Let us now prove $(a)$. Let $\left(m_{n}\right)_{n}$ be a sequence in $\mathcal{C}$ such that $m_{n} \rightarrow m$ in the $C\left([0, T] ; \mathcal{P}_{1}\left(\mathbb{R}^{2 N}\right)\right)$ topology. We want to prove that $\mathcal{T}\left(m_{n}\right) \rightarrow \mathcal{T}(m)$ in $C\left([0, T] ; \mathcal{P}_{1}\left(\mathbb{R}^{2 N}\right)\right)$. We observe that hypothesis (H3) ensures that the functions $(x, v, t) \mapsto F\left[m_{n}(t)\right](x, v)$ and $(x, v) \mapsto G\left[m_{n}(T)\right](x, v)$ converge locally uniformly to the map $(x, v, t) \mapsto F[m(t)](x, v)$ and respectively $(x, v) \mapsto G[m(T)](x, v)$. Moreover, Lemma 3.2 entails that the solutions $u_{n}$ to problem (3.4) with $\bar{m}=m_{n}$ are locally uniformly bounded and locally uniformly Lipschitz continuous. Therefore, by standard stability results for viscosity solutions, the sequence $\left(u_{n}\right)_{n}$ converges locally uniformly to viscosity the solution $u$ to problem (3.4) with $\bar{m}=m$. Moreover, from Lemma 3.3, the functions $u_{n}$ are uniformly semi-concave; hence, by [11, Theorem 3.3.3], $D u_{n}$ converge a.e. to $D u$.

By Proposition 4.1 and Remark 4.1, the function $\mathcal{T}\left(m_{n}\right)$ verifies the bounds in Lemma 4.3 with a constant $K$ independent of $n$. Hence, the sequence $\left(\mathcal{T}\left(m_{n}\right)\right)_{n}$ is uniformly bounded in $C\left([0, T] ; \mathcal{P}_{1}\left(\mathbb{R}^{2 N}\right)\right.$ ) (by Lemma 4.3-(3) and Remark 4.1, and because the subsets of $\mathcal{P}_{1}\left(\mathbb{R}^{2 N}\right)$ whose elements have uniformly bounded second moment are relatively compact in $\mathcal{P}_{1}\left(\mathbb{R}^{2 N}\right)$ ), and uniformly Hölder continuous in time with values in $\mathcal{P}_{1}\left(\mathbb{R}^{2 N}\right)$ (by Lemma 4.3-(2) and Remark 4.1). Therefore, by Ascoli-Arzelà and 
Banach-Alaoglu theorems, there exists a subsequence $\left(\mathcal{T}\left(m_{n_{k}}\right)\right)_{k}$ which converges to some $\mu \in C\left([0, T] ; \mathcal{P}_{1}\left(\mathbb{R}^{2 N}\right)\right)$ in the $C\left([0, T] ; \mathcal{P}_{1}\left(\mathbb{R}^{2 N}\right)\right)$-topology and in the $L_{\text {loc }}^{\infty}\left((0, T) \times \mathbb{R}^{2 N}\right)$ weak-* topology. As in Remark 4.1, $\mu$ verifies the bounds in Lemma 4.3 and $\mu(0)=m_{0}$.

Observe that $\mathcal{T}\left(m_{n_{k}}\right)$ solves problem (4.1) with $u$ replaced by $u_{n_{k}}$,

$$
\int_{0}^{T} \int_{\mathbb{R}^{2 N}} \mathcal{T}\left(m_{n_{k}}\right)\left(-\partial_{t} \psi+D_{v} \psi \cdot D_{v} u_{n_{k}}-v \cdot D_{x} \psi\right) d x d v d t=0
$$

for any $\psi \in C_{c}^{\infty}\left((0, T) \times \mathbb{R}^{2 N}\right)$. Passing to the limit as $k \rightarrow \infty$, we get that $\mu$ is a solution to (4.1). By the uniqueness result established in Proposition 4.2, we deduce that $\mu=\mathcal{T}(m)$, and that the whole sequence $\left(\mathcal{T}\left(m_{n}\right)\right)_{n}$ converges to $\mathcal{T}(m)$.

Let us now prove $(b)$; since $\mathcal{C}$ is closed, it is enough to prove that $\mathcal{T}(\mathcal{C})$ is a precompact subset of $C\left([0, T] ; \mathcal{P}_{1}\left(\mathbb{R}^{2 N}\right)\right)$. Let $\left(\mu_{n}\right)_{n}$ be a sequence in $\mathcal{T}(\mathcal{C})$ with $\mu_{n}=\mathcal{T}\left(m_{n}\right)$ for some $m_{n} \in \mathcal{C}$; we wish to prove that, possibly for a subsequence, $\mu_{n}$ converges to some $\mu$ in the $C\left([0, T] ; \mathcal{P}_{1}\left(\mathbb{R}^{2 N}\right)\right)$-topology as $n \rightarrow \infty$.

By Remark 4.1, the functions $\mathcal{T}\left(m_{n}\right)$ satisfy the estimates in Lemma 4.3 with the same constant $K$. Since the subsets of $\mathcal{P}_{1}\left(\mathbb{R}^{2 N}\right)$ whose elements have uniformly bounded second moment are relatively compact in $\mathcal{P}_{1}\left(\mathbb{R}^{2 N}\right)$, Lemma $4.3-(3)$ ensures that the sequence $\left(\mathcal{T}\left(m_{n}\right)\right)_{n}$ is uniformly bounded. Moreover, Lemma 4.3-(2) yields that the sequence $\left(\mathcal{T}\left(m_{n}\right)\right)_{n}$ is uniformly bounded in $C^{1 / 2}\left([0, T] ; \mathcal{P}_{1}\left(\mathbb{R}^{2 N}\right)\right)$ and $L^{\infty}\left(0, T ; \mathcal{P}_{2}\left(\mathbb{R}^{2 N}\right)\right)$. By arguing as in the proof of Proposition 4.1, we obtain that, possibly for a subsequence (still denoted by $\left.\mathcal{T}\left(m_{n}\right)\right), \mathcal{T}\left(m_{n}\right)$ converges to some $\mu$ in the $C\left([0, T] ; \mathcal{P}_{1}\left(\mathbb{R}^{2 N}\right)\right)$-topology.

2. Theorem 4.1 ensures that, if $(u, m)$ is a solution of $(2.1)$, for any function $\psi \in$ $C_{b}^{0}\left(\mathbb{R}^{2 N}\right)$

$$
\int_{\mathbb{R}^{2 N}} \psi(x, v) m(x, v, t) d x d v=\int_{\mathbb{R}^{2 N}} \psi\left(\bar{\gamma}_{x, v}(t)\right) m_{0}(x, v) d x d v
$$

where $\bar{\gamma}_{x, v}$ is the solution of $(2.2)$ (uniquely defined for a.e. $(x, v) \in \mathbb{R}^{2 N}$ ).

Proof of Proposition 2.1. Let $\left(u_{1}, m_{1}\right)$ and $\left(u_{2}, m_{2}\right)$ be two solutions to system (2.1) in the sense of Definition 2.1. By Theorem 4.1, for $i=1,2$, the function $m_{i}$ satisfies (4.2) with $\bar{\gamma}_{x, v}$ replaced by $\bar{\gamma}_{x, v}^{i}$, which for a.e. $(x, v)$ is the solution to $(2.2)$ with $u$ replaced by $u_{i}$.

Moreover, let us recall from Lemma 3.2-(1) that the Lipschitz constant of $u_{i}$ has an at most linear growth in $v$. By Gronwall's Lemma we obtain that $\bar{\gamma}_{x, v}^{i}$ is bounded. Since $m_{0}$ has compact support, we deduce the function $m_{i}$ has compact support. In particular, we obtain that $\bar{u}:=u_{1}-u_{2}$ is an admissible test-function for the continuity equation satisfied by $m_{i}$.

Taking advantage of the convexity of our Hamiltonian $\mathcal{H}$ and of the monotonicity of the couplings $F$ and $G$, we can conclude the proof following the same arguments as in [26, Theorem 2.5].

\section{Appendix}

Let us now consider the second order MFG system: for a positive number $\sigma$,

$$
\begin{cases}(i)-\partial_{t} u-\sigma \Delta_{x, v} u-v \cdot D_{x} u+\frac{1}{2}\left|D_{v} u\right|^{2}-\frac{1}{2}|v|^{2}-l(x, v)=F[m](x, v), & \text { in } \mathbb{R}^{2 N} \times(0, T), \\ (i i) \partial_{t} m-\sigma \Delta_{x, v} m-\operatorname{div}_{v}\left(m D_{v} u\right)-v \cdot D_{x} m=0, & \text { in } \mathbb{R}^{2 N} \times(0, T), \\ (i i i) m(x, v, 0)=m_{0}(x, v), \quad u(x, v, T)=G[m(T)](x, v), & \text { on } \mathbb{R}^{2 N} .\end{cases}
$$


We aim at proving the existence and uniqueness of a classical solution to system (6.1). We shall see that these results are byproducts of the estimates that we have already used above in the vanishing viscosity limit. More precisely, the properties obtained in Section 4 will play a crucial role in what follows.

Theorem 6.1. Under our standing assumptions, there exists a classical solution to problem (6.1). Moreover, if the coupling costs $F$ and $G$ satisfy (2.3), the solution is unique.

Proof. Our arguments are reminiscent of those used in the proof of [12, Theorem 3.1]. We introduce

$$
\mathcal{C}:=\left\{m \in C^{0}\left([0, T] ; \mathcal{P}_{1}\left(\mathbb{R}^{2 N}\right)\right): \quad m(0)=m_{0}\right\}
$$

which is a non-empty closed and convex subset of $C^{0}\left([0, T] ; \mathcal{P}_{1}\left(\mathbb{R}^{2 N}\right)\right)$. We define a map $\mathcal{T}$ as follows: for any $m \in \mathcal{C}$, let $u$ be the unique solution to $(6.1)-(i)$ and $u(x, v, T)=$ $G[m(T)](x, v)$ found in Lemma 4.1; we set $\mathcal{T}(m)=\mu$ where $\mu$ is the unique solution to (6.1)-(ii) and $m(x, v, 0)=m_{0}(x, v)$ found in Lemma 4.2. Lemma 4.3 ensures that $\mathcal{T}$ maps $\mathcal{C}$ into itself.

By the same arguments as in the proof of Theorem 2.1, the map $\mathcal{T}$ is continuous with respect to the norm of $C\left([0, T] ; \mathcal{P}_{1}\left(\mathbb{R}^{2 N}\right)\right)$ and it is compact. Hence, Schauder fixed point theorem ensures the existence of a fixed point $m$ for $\mathcal{T}$. Let $u$ denote the corresponding solution to (6.1)-(i) and -(iii). By Lemma 4.1 and Lemma 4.2 again, $u$ and $m$ are regular. In conclusion, $(u, m)$ is the desired solution to $(6.1)$.

Let us now prove the uniqueness part of the statement. Let $\left(u_{1}, m_{1}\right)$ and $\left(u_{2}, m_{2}\right)$ be two solutions; set $\bar{u}=u_{1}-u_{2}$. Our aim is to follow the arguments in the proof of Proposition 2.1. To this end, it is enough to prove that $\bar{u}$ is an admissible test-function for $m_{1}$ and $m_{2}$. Indeed, for any $R>1$, let $\phi_{R}$ be a cut-off function in $\mathbb{R}^{2 N}$ defined by $\phi_{R}(x, v):=\phi_{1}(x / R, v / R)$ where $\phi_{1}$ is a $C^{2}$ function such that $\phi_{1}=1 \mathrm{in} B_{1}, \phi_{1}=0$ outside $B_{2}$. Clearly,

$$
D_{x, v} \phi_{R}=0 \quad \text { outside } \overline{B_{2 R} \backslash B_{R}}, \quad\left\|D_{x, v} \phi_{R}\right\|_{\infty} \leqslant C / R \quad \text { and }\left\|\Delta_{x, v} \phi_{R}\right\|_{\infty} \leqslant C / R^{2} .
$$

Using $\phi_{R} \bar{u}$ as test-function in (6.1)-(ii) with $(u, m)=\left(u_{i}, m_{i}\right)$ for $i=1$ or $i=2$, we get

$$
\begin{aligned}
& 0=\quad \iint_{R^{2 N} \times[0, T]} m\left[-\phi_{R} \partial_{t} \bar{u}-\sigma \Delta_{x, v}\left(\phi_{R} \bar{u}\right)+D_{v} u \cdot D_{v}\left(\phi_{R} \bar{u}\right)+v \cdot D_{x}\left(\phi_{R} \bar{u}\right)\right] d x d v d t \\
& \quad+\int_{\mathbb{R}^{2 N}} m(x, v, T) \phi_{R}\left(G\left[m_{1}(T)\right](x, v)-G\left[m_{2}(T)\right](x, v)\right) d x d v-\int_{\mathbb{R}^{2 N}} m_{0} \phi_{R} \bar{u} d x d v \\
& =\quad \iint_{R^{2 N} \times[0, T]} m \phi_{R}\left(2 v \cdot D_{x} \bar{u}+\frac{\left|D_{v} u_{2}\right|^{2}-\left|D_{v} u_{1}\right|^{2}}{2}+F\left[m_{1}\right]-F\left[m_{2}\right]+D_{v} u \cdot D_{v} \bar{u}\right) d x d v d t \\
& \quad+\int_{\mathbb{R}^{2 N}} m(x, v, T) \phi_{R}\left(G\left[m_{1}(T)\right](x, v)-G\left[m_{2}(T)\right](x, v)\right) d x d v-\int_{\mathbb{R}^{2 N}} m_{0} \phi_{R} \bar{u} d x d v \\
& \quad+\quad \iint_{R^{2 N} \times[0, T]} m\left(-\sigma \bar{u} \Delta_{x, v} \phi_{R}-2 \sigma D_{x, v} \phi_{R} \cdot D_{x, v} \bar{u}\right) d x d v d t \\
& \quad \int_{R^{2 N} \times[0, T]} m\left(\bar{u} D_{v} u \cdot D_{v} \phi_{R}+\bar{u} v \cdot D_{x} \phi_{R}\right) d x d v d t
\end{aligned}
$$

where the second equality is due to equation (6.1)-(i). Since $m>0$ and $m \in L^{\infty}\left((0, T) ; \mathcal{P}_{2}\left(\mathbb{R}^{2 N}\right)\right)$ (see Lemma 4.2 and Lemma 4.3), by the estimates on $u_{i}$ in Lemma 4.1, the dominated 
convergence theorem ensures that as $R \rightarrow \infty$, the first two lines in right hand side of $(6.3)$ converge to

$$
\begin{aligned}
\iint_{R^{2 N} \times[0, T]} m & {\left[2 v \cdot D_{x} \bar{u}-\frac{\left|D_{v} u_{1}\right|^{2}}{2}+\frac{\left|D_{v} u_{2}\right|^{2}}{2}+F\left[m_{1}\right]-F\left[m_{2}\right]+D_{v} u \cdot D_{v} \bar{u}\right] d x d v d t } \\
+ & \int_{\mathbb{R}^{2 N}} m(x, v, T)\left(G\left[m_{1}(T)\right](x, v)-G\left[m_{2}(T)\right](x, v)\right) d x d v-\int_{\mathbb{R}^{2 N}} m_{0} \bar{u} d x d v ;
\end{aligned}
$$

hence, it remains to prove that the last two lines in the right hand side of (6.3) converge to 0 . Indeed, again by Lemmas 4.1, 4.2 and 4.3, and by our estimates (6.2), the dominated convergence theorem yields

$$
\iint_{R^{2 N} \times[0, T]} m\left(-\sigma \bar{u} \Delta_{x, v} \phi_{R}-2 \sigma D_{x, v} \phi_{R} \cdot D_{x, v} \bar{u}\right) d x d v d t \rightarrow 0 .
$$

Let us now address to the last integral in the right hand side of (6.3): the properties in (6.2) entail

$$
\left|m\left(\bar{u} D_{v} u \cdot D_{v} \phi_{R}+\bar{u} v \cdot D_{x} \phi_{R}\right)\right| \leqslant C m\left(1+|v|^{2}\right) \chi_{R}
$$

where $\chi_{R}$ is the characteristic function of $B_{2 R} \backslash B_{R}$. Moreover, since $m \in L^{\infty}\left((0, T) ; \mathcal{P}_{2}\left(\mathbb{R}^{2 N}\right)\right)$, the right hand side in the last inequality belongs to $L^{1}$ independently of $R$. Therefore, again by the dominated convergence theorem, we get that as $R \rightarrow \infty$ the last integral in (6.3) converges to 0.

Acknowledgment. The authors are grateful to the anonymous referees for their fruitful comments and suggestions. The work of Y. Achdou and N. Tchou was partially supported by the ANR (Agence Nationale de la Recherche) through MFG project ANR-16-CE400015-01. Y. Achdou acknowledges support from the Chair Finance \& Sustainable Development and the FiME Lab (Institut Europlace de Finance). The work of N. Tchou is partially supported by the Centre Henri Lebesgue ANR-11-LABX-0020-01. P. Mannucci and C. Marchi are members of GNAMPA-INdAM and were partially supported also by the research project of the University of Padova "Mean-Field Games and Nonlinear PDEs" and by the Fondazione CaRiPaRo Project "Nonlinear Partial Differential Equations: Asymptotic Problems and Mean-Field Games".

\section{References}

[1] Y. Achdou, F. J. Buera, J-M. Lasry, P-L. Lions, B. Moll, Partial differential equation models in macroeconomics, Fill's. Trans. R. Soc. Lond. Ser. A Math. Phys. Eng. Sci., 372 (2014) 2028, 20130397, 19 pp.

[2] L. Ambrosio, N. Gigli, G. Savaré, Gradient flows in metric spaces and in the space of probability measures, Lectures in Mathematics ETH Zürich. Birkhaüser Verlag, Basel 2005.

[3] J-D. Benamou, G. Carlier, F. Santambrogio, Variational mean field games, Active particles. Vol. 1, Advances in theory, models, and applications, 141-171, Model. Simul. Sci. Eng. Technol., Birkhäuser/Springer, Cham, 2017. 
[4] M. Bardi, I. Capuzzo Dolcetta, Optimal control and viscosity solutions of Hamilton-Jacobi Bel lman equations, Systems and Control: Foundations and Applications. Birkhauser, Boston 1997.

[5] V. I. Bogachev, N.V. Krylov, M. Röckner, S. V. Shaposhnikov, FokkerPlanck-Kolmogorov equations, Mathematical Surveys and Monographs, 207. American Mathematical Society, Providence, RI, 2015.

[6] R. Buckdahn, P. Cannarsa, M. Quincampoix, Lipschitz continuity and semiconcavity properties of the value function of a stochastic control problem, NoDEA Nonlinear Differential Equations Appl. 17 (2010), no. 6, 715-728.

[7] P. Cannarsa, R. Capuani, Existence and uniqueness for Mean Field Games with state constraints, PDE models for multi-agent phenomena, 49-71, Springer INdAM Ser., 28, Springer, Cham, 2018.

[8] P. Cannarsa, R. Capuani, P. Cardaliaguet, $C^{1,1}$-smoothness of constrained solutions in the calculus of variations with application to mean field games, Math. Eng. 1 (2018), no. 1, 174-203.

[9] P. Cannarsa, R. Capunini, P. Cardaliaguet, Mean Field Games with state constraints: from mild to pointwise solutions of the PDE system, avaliable at https://arxiv.org/abs/1812.11374.

[10] P. Cannarsa, C. Mendico, Mild and weak solutions of Mean Field Games problem for linear control systems, avaliable at https://arxiv.org/abs/1907.02654

[11] P. Cannarsa, C. Sinestrari, Semi-Concave Functions, Hamilton-Jacobi Equations, and Optimal Control, Progress in Nonlinear Differential Equations and Their Applications, 48, Birkhauser, Boston 2004.

[12] P. Cardaliaguet, Notes on Mean Field Games, from P.L. Lions lectures at College de France (2012), available at https://www.ceremade.dauphine.fr/ cardalia/MFG20130420.pdf.

[13] P. Cardaliaguet, Long time average of first order mean field games and weak KAM theory, Dyn. Games Appl. 3 (2013), 473-488.

[14] P. Cardaliaguet, Weak solutions for first order mean field games with local coupling, Analysis and geometry in control theory and its applications, 111-158, Springer INdAM Ser., 11, Springer, Cham, 2015.

[15] P. Cardaliaguet, S. Hadikhanloo, Learning in mean field games: the fictitious play, ESAIM Control Optim. Calc. Var. 23 (2017), no. 2, 569-591.

[16] P. Cardaliaguet, A. Mészáros, F. Santambrogio, First order mean field games with density constraints: pressure equals price, SIAM J. Control Optim. 54 (2016), no. 5, 2672-2709.

[17] F. Clarke, Optimization and nonsmooth analysis, Classics in Applied Mathematics 5, SIAM, Philadelphia, PA, 1990 (2nd edition). 
[18] F. Clarke, Functional Analysis, Calculus of Variations and Optimal Control , Graduate Text in Mathematics 264, Springer-Verlag, London 2013.

[19] F. DA Lio, O. Ley, Uniqueness results for second-order Bellman-Isaacs equations under quadratic growth assumptions and applications, SIAM J. Control Optim. 45 (2006), no. 1, 74-106.

[20] I. Karatzas, S.E. Shreve, Brownian motion and stochastic calculus, Second edition. Graduate Texts in Mathematics, 113. Springer-Verlag, New York, 1991.

[21] Y. IKEDA, The Cauchy problem of linear parabolic equations with discontinuous and unbounded coefficients, Nagoya Math. J. 41 (1971), 33-42.

[22] H. IsHII, On the equivalence of two notions of weak solutions, viscosity solutions and distribution solutions Funkcial. Ekvac. 38 (1995), no. 1, 101-120.

[23] O. A. Lady ženskaja, V. A. Solonnikov, N. N. Ural'Ceva, Linear and quasilinear equations of parabolic type. Translated from the Russian by S. Smith. Translations of Mathematical Monographs, Vol. 23 American Mathematical Society, Providence, R.I. 1968.

[24] J-M. Lasry And P-L. Lions, Jeux à champ moyen. I. Le cas stationnaire, $C . R$. Math. Acad. Sci. Paris, 343 (2006), no. 9, 619-625.

[25] J-M. Lasry And P-L. Lions, Jeux à champ moyen. II. Horizon fini et contrôle optimal, C. R. Math. Acad. Sci. Paris, 343 (2006), no. 10, 679-684.

[26] J-M. Lasry And P-L. Lions, Mean field games, Jpn. J. Math., 2 (2007), no. 1, 229-260.

[27] G.M. Lieberman, Second order parabolic differential equations, World Scientific Publishing Co., Inc., River Edge, NJ, 1996.

[28] P-L. Lions, Cours au Collège de France. www.college-de-france.fr.

[29] P. Mannucci, C. Marchi, C. Mariconda, N. Tchou, Non-coercive first order Mean Field Games, J. Differential Equations 269 (2020), no. 5, 4503-4543.

[30] C. Orrieri, A. Porretta, G. Savaré, A variational approach to the mean field planning problem, J. Funct. Anal. 277 (2019), no. 6, 1868-1957.

[31] C. Villani, Optimal transport, old and new, Springer-Verlag, Berlin 2009. 